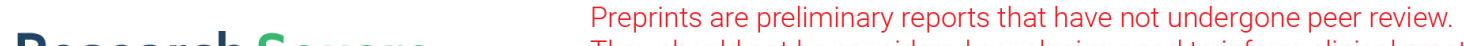 They should not be considered conclusive, used to inform clinical practice, or referenced by the media as validated information. \\ Theoretical Analysis and Experimental Research of Surface Texture Hydrodynamic Lubrication
}

\section{Dan Li}

university of Jinan

Xuefeng YANG ( $\square$ me_yangxf@ujn.edu.cn )

College of Mechanical Engineering, University of Jinan, Jinan 250022, China

Wu Yuanbo

university of Jinan

Cheng Jian

hubei university of technology

Wang Shouren

university of jinan

Wan Zhuang

university of jinan

Liu Wenbo

university of jinan

Xia Guofeng

university of jinan

\section{Original Article}

Keywords: Hydrodynamic lubrication, Tribological characteristics, Surface texture

Posted Date: September 22nd, 2020

DOI: https://doi.org/10.21203/rs.3.rs-78890/v1

License: (1) (i) This work is licensed under a Creative Commons Attribution 4.0 International License.

Read Full License

Version of Record: A version of this preprint was published at Chinese Journal of Mechanical Engineering on April 5th, 2022. See the published version at https://doi.org/10.1186/s10033-022-00691-7. 


\title{
Theoretical Analysis and Experimental Research of Surface Texture Hydrodynamic Lubrication
}

\author{
Li Dan, Yang Xuefeng ${ }^{\mathrm{a}, *}$, Wu Yuanbo ${ }^{\mathrm{a}}$, Cheng Jian ${ }^{\mathrm{b}}$, Wang Shouren ${ }^{\mathrm{a}}$, Wan Zhuang ${ }^{\mathrm{a}}$, \\ Liu Wenbo ${ }^{a}$, Xia Guofeng ${ }^{a}$ \\ ${ }^{a}$ College of Mechanical Engineering, University of Jinan, Jinan 250022, China \\ ${ }^{b}$ College of Mechanical Engineering, Hubei University of Technology, Hubei 430068, China
}

Abstract: This paper established a mathematical model of hydrodynamic lubrication through theoretical analysis, solved the key variables of the mathematical model through simulation and validated the mathematical model using the experimental results. The purpose is to summarize a set of surface texture theory and optimal selection equations for complex working conditions. After comparing the experimental results with the results obtained by the mathematical model, it is found that the two are basically the same in the ranking of the anti-friction properties of different textures, and there is an error of $10 \%-40 \%$ in the friction coefficient value.

Keywords: Hydrodynamic lubrication; Tribological characteristics; Surface texture

\section{Introduction}

As an effective means of reducing friction, texture has a very broad industrial application prospect. The working mechanism of texture [1] is the theoretical basis of its optimal design. Many studies usually comprehensively analyze the tribological theory and test results, and continuously improve the design of the texture [2-5] to make it more adaptable.

Ryk et al. [6] found that the friction force of the laser-processed surface textured cylinder liner piston ring was reduced by $25 \%$. This is because the surface texture enhances the 
1 hydrodynamic lubrication effect under high-speed reciprocating motion. Etsion I et al. [7]

2 constructed a mathematical model for the performance analysis of laser-machined

3 microtextures on the surface of mechanical seals, using liquid film pressure as the main

4 design parameter. The results show that the ratio of hole depth to hole diameter has a very

5 significant effect on pressure. And there is an optimal parameter value that increases friction

6 performance. Wakuda Manabu et al. [8] found that there is a certain size and area ratio to

7 optimize the friction characteristics of the friction pair, while the shape parameters of the

8 texture have little effect on the friction performance. Tonder.K [9] believes that the surface

9 texture can store lubricant in the inlet area. Therefore, the rigidity and damping of the

10 lubricating oil film can be improved, thereby improving the stability of the oil film. Fowell M

11 et al. [10] proposed a new fluid dynamic pressure lubrication mechanism. The pressure

12 difference between the entrance of the texture and the inside of the texture is used to "suck"

13 the lubricating oil into the inside of the texture to form an additional hydrodynamic pressure.

14 This mechanism is considered to be the reason for promoting lubricant film formation and

15 reducing friction and wear on the surface. Hoppermann et al. [11] found that texturing on both

16 friction surfaces would increase friction. Only a texturing process on one surface will reduce

17 friction.

Krupka.I et al. [12,13] explored the effect of micro-pit morphology on the thickness of the oil film. The experiment used colorimetric interference method to observe the change of lubricating film thickness. The study found that the micro-pit shape can increase the local minimum oil film thickness. Rahmani et al. [14] used an optimization program to find the best dimensional parameters to increase the pressure-bearing capacity and lubricant flow ratio of the asymmetrically textured slider bearing and reduce the friction coefficient. Charitopoulos 
1 et al. [15] found that textures of different shapes and sizes show different improvements on

2 the friction resistance of friction surfaces. Andersson et al. [16] found that the texture with

3 low density and large depth-to-diameter ratio combined with high viscosity lubricating oil can

4 increase the anti-friction properties. Kligerman et al. [17] studied the hydrodynamic effects of

5 laser surface texture in sealed micropores through a finite element model. Compared with the

6 smooth surface, the surface texture can effectively reduce the friction torque and improve the

7 tribological performance of the air seal. Suh et al. [18] used a dynamic model to study the

8 effect of surface texture of the slider air bearing on adhesion and friction. The simulation

9 results show that the surface texture reduces the adhesion and friction by reducing the 10 effective contact area between the slider and the medium surface and preferentially 11 roughening the interface.

12 Lubricating conditions can affect the anti-friction mechanism of texture [19-21]. The 13 friction wear test results alone cannot explain the working mechanism of the surface texture under a certain lubrication condition. The lubrication state of the contact surface is closely related to the oil parameters [22-25]. Thickness of oil film and surface roughness can be used to determine the lubrication state under different operating conditions. The influence of surface texture on the thickness of lubricating oil film should be studied emphatically. working conditions were summarized. By studying the surface texture and tribological characteristics of different morphology and size features, according to the working conditions of the moving friction pair, the optimal surface texture can be solved and manufactured.

\section{Establishment of mathematical model}


1 of hydrodynamic lubrication of different surface textures was established, and the solution

2 method of the optimal surface texture model was determined. The purpose is to study how to

3 choose the optimal solution for different surface textures under different working conditions,

4 and to provide theoretical guidance for subsequent research.

\section{$5 \quad 2.1$ Introduction to basic theory}

\section{$6 \quad$ 2.1.1 Derivation of $\mathrm{N}-\mathrm{S}$ equation}

The N-S equation is obtained by substituting and sorting the components of the viscous

8 fluid motion momentum equation in the form of stress with the expression given by the generalized Newton's internal friction law [26]. If the fluid is incompressible, the dynamic viscosity $\mu$ is constant. The N-S equation can be simplified as:

$$
\text { In the formula, } \nabla^{2}=\frac{\partial^{2}}{\partial x^{2}}+\frac{\partial^{2}}{\partial y^{2}}+\frac{\partial^{2}}{\partial z^{2}}
$$
forms of the N-S equation of incompressible viscous flow can be obtained from the relationship of dynamic viscosity $\mu$, kinematic viscosity $v$ and density $\rho$.

$$
\begin{aligned}
& \frac{d v_{x}}{d t}=f_{x}-\frac{1}{\rho} \frac{\partial p}{\partial x}+v \nabla^{2} v_{x} \\
& \frac{d v_{y}}{d t}=f_{y}-\frac{1}{\rho} \frac{\partial p}{\partial \mathrm{y}}+v \nabla v_{y} \\
& \frac{d v_{z}}{d t}=f_{z}-\frac{1}{\rho} \frac{\partial p}{\partial \mathrm{z}}+v \nabla v_{z}
\end{aligned}
$$

\subsubsection{Couette flow}


The N-S equation of viscous fluid motion is a second-order nonlinear partial differential equation, which is difficult to solve. In addition, in practical engineering, the viscous flow with complex flow boundaries is encountered, and with the change of time and space, the flow parameters in the flow field are also constantly affected and changed. But when the flow boundary is relatively simple and the flow parameters are mostly constant, the solution of the flow can be obtained. As a kind of viscous flow, Couette flow is of great significance in engineering, and accurate analytical solutions can be obtained under some conditions [27].

The typical form of Couette flow is two infinite planes with a distance of $h_{0}$, in which an incompressible fluid with a dynamic viscosity of $\mu$ flows in a fixed direction. Couette flow also includes two other forms. The first is that there is a speed difference between the two planes, so that the two planes produce relative motion but the pressure in the direction of flow does not change. The second is that there is not only a speed difference between the two planes, but also a pressure gradient in the flow direction. At this time, the solution of the linear equation is additive, and the independent solutions of velocity and pressure can be added.

\subsection{Basic derivation of theory}

Each surface texture corresponds to one or more pressure values and velocity values that can give full play to its tribological properties. This matching mechanism is the core of theory. Before deriving the basic theory, the applicable premise must be determined first:

(1) The effects of volume force such as gravity and magnetic force are ignored.

(2) Assume that there is no relative sliding between the fluid and solid interface.

(3) The fluid belongs to a kind of Couette flow, but it is not exactly the same.

(4) The fluid is Newtonian fluid, and the flow mode is turbulent flow. There may be 
1 vortex and turbulent flow in the oil film.

(5) Compared with the viscous force of lubricating oil, the inertial force is smaller, so its

3 effect is ignored, including the inertial force when the fluid accelerates and the centrifugal

4 force when the fluid moves in a circular motion.

\section{$5 \quad$ 2.2.1 Setting of fluid boundary conditions based on Couette flow}

As shown in Figure 1, the thickness of the oil film between the two moving friction pairs

7 is $h_{0}$. The lubricating oil is an incompressible fluid with a dynamic viscosity of $\mu$, which is

8 driven by the upper plate to flow positively towards the $\mathrm{X}$ axis, and the moving speed of the

9 upper plate is $U$. The depth of texture is $h_{\mathrm{p}}$, and the cross-sectional width is $2 r_{\mathrm{p}}$. The upper 10 plate moves to the right at speed $U$, while the lubricating oil has a pressure gradient in the $\mathrm{x}$ 11 direction. The red line represents the speed gradient change. The purple line represents the 12 velocity distribution under different pressures. When $P>0$, the velocity distribution is the rightmost purple line, the pressure promotes the flow of lubricating oil, and the average 14 velocity is greater than the velocity without pressure difference. When $P<0$, the velocity distribution is the leftmost purple line. At this time, the flow caused by the upper plate is not enough to overcome the flow caused by the reverse pressure difference, so reverse flow occurs.

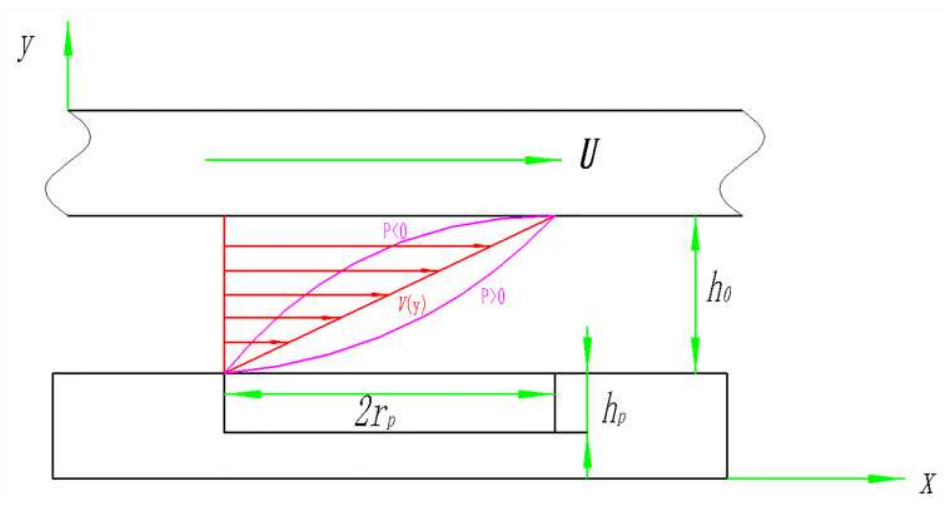

Figure 1. Couette flow 
2

$$
4 \quad \frac{v_{x}(y)}{y}=U \frac{1}{h_{0}}-\frac{1}{2 \mu} \frac{d p}{d x}\left(h_{0}-y\right)
$$

$$
v_{x}(y)=U \frac{y}{h_{0}}-\frac{1}{2 \mu} \frac{d p}{d x} y\left(h_{0}-y\right)
$$

\subsubsection{Summary of theory formula} Equation 6 is obtained.

By dividing both sides of Equation 3 by $y$, the equation becomes:

Equation 4 is the relationship between the fluid velocity and pressure in the Couette flow form. This equation is a simplified N-S equation based on Couette flow.

Substituting Equation 4 into the pressure-friction conversion equation (Equation 5),

$$
\begin{aligned}
& f=\frac{\frac{d p}{d x \cdot\left(D_{1}+D_{2}\right)}\left(D_{1}^{2}-D_{2}{ }^{2}\right) \pi}{n}=\frac{\frac{d p}{d x}}{n}\left(D_{1}-D_{2}\right) \pi \\
& f=\frac{\frac{U}{h_{0}}-\frac{v_{x}(y)}{y}}{\left(h_{0}-y\right)} \cdot \frac{2 \mu}{n} \cdot\left(D_{1}-D_{2}\right) \pi
\end{aligned}
$$

The conversion equation 5 is derived from the actual experimental conditions in this paper. $\mathrm{d} p / \mathrm{d} x \cdot\left(D_{1}-D_{2}\right) / \mathrm{n}$ is the pressure value in the unit area, $n$ is the number of texture distribution on the surface of the test piece, $D_{1}$ is the inner diameter of the grinding piece, $D_{2}$ is the outer diameter of the grinding piece. Equation 5 can be used to combine the hydrodynamic equations with the surface texture parameters, and fully fit the actual working conditions of this article, which improves the accuracy and credibility of the equation. However, there is a certain error in the solution of the pressure in the cell texture area, and this equation also has a certain error. Equation 6 is the relationship between friction force and fluid flow characteristics, it is necessary to continue to derive the equation. The final equation 
17 is obtained.

$2 \quad \frac{F}{f}=\frac{P \cdot S}{f}=\frac{P \cdot S\left(h_{0}-y\right) \cdot n}{\left(\frac{U}{h_{0}}-\frac{v_{x}(y)}{y}\right) \cdot 2 \mu\left(D_{1}-D_{2}\right) \pi}$

3

4

5

6

7

8

9

\subsection{Simplification of theory formula}

According to the experimental conditions in this paper, the substitution amount is simplified. The outer diameter, inner diameter and contact area of the grinding piece are fixed. The lubricating oil is selected from Mobil Series No. 1 lubricating oil, and the parameters are fixed. In Equation 7, the value of $h_{0}$ is critical. Since there is an independent variable y in the film thickness direction, if $h_{0}$ is retained, it will increase the difficulty of solving and make the equation have multiple solutions. The value of $h_{0}$ is set to 1 , and the range of the independent variable $\mathrm{y}$ is $[0,1]$, and Equation 8 is obtained.

$$
u=\frac{P \cdot 0.0000885 \cdot(1-y) \cdot 24}{\left(\frac{U}{1}-\frac{v_{x}(y)}{y}\right) \cdot 2 \cdot 0.055 \cdot(0.01+0.014) \pi}=\frac{P \cdot(1-y) \cdot 0.002124}{0.0083 \cdot\left(\frac{U}{1}-\frac{v_{x}(y)}{y}\right)}
$$

In Equation 8, the pressure $P$ and speed $U$ need to be set according to the actual working

conditions. $\frac{v_{x}(y)}{y}$ is the first-order partial derivative of the flow speed of the lubricating oil in the $\mathrm{X}$-axis direction. In this model, the velocity $v$ at any point has only the $\mathrm{x}$-axis component, $v_{\mathrm{y}}=v_{\mathrm{z}}=0$, so it can be considered that $v_{\mathrm{x}}=\mathrm{v}$. This part needs to obtain data through simulation to solve, the solution process is explained in detail in the simulation analysis section.

\section{Simulation analysis}

The FLUENT module in ANSYS software was used to simulate the hydrodynamic lubrication effect. The purpose is to obtain data that cannot be obtained under experimental conditions to provide data support for the model solution.

\subsection{The process of simulation analysis}

In this paper, four kinds of surface textures are selected as the research object, including 
1 groove texture, composite texture, chevron texture and circular texture. The groove texture

2 and the circular texture are typical textures. As new types of texture, chevron texture and

3 composite texture have great research value. The upper sample is 45 steel with an inner

4 diameter of $10 \mathrm{~mm}$ and an outer diameter of $14.6 \mathrm{~mm}$, and the lower sample is a round YT15

5 cemented carbide, as shown in Figure 2. The texture size is shown in Figure 3.

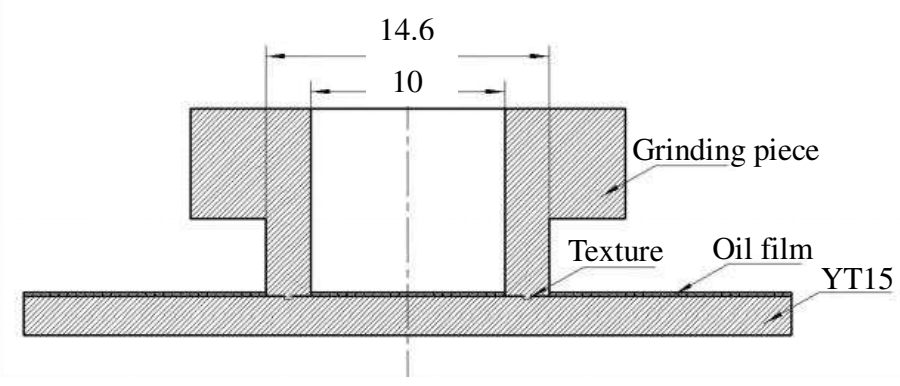

Figure 2. Schematic diagram of experiment

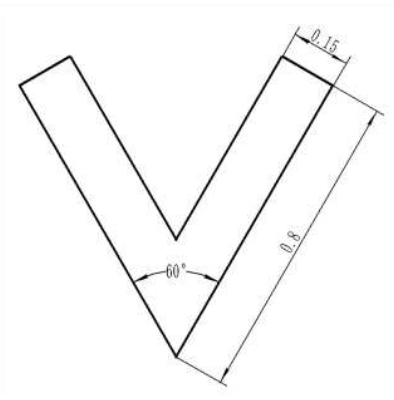

(a) chevron texture

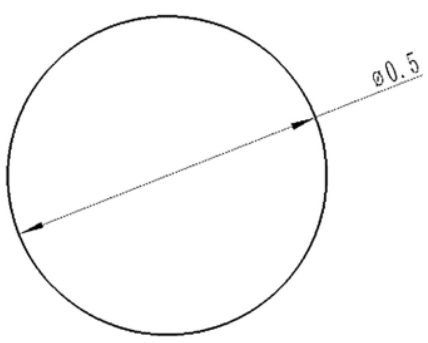

(c) circular texture

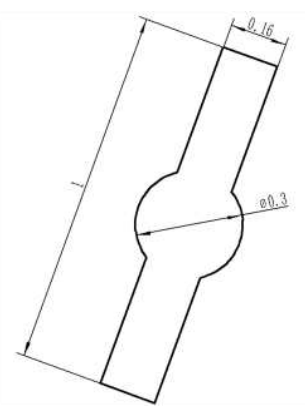

(b) composite texture

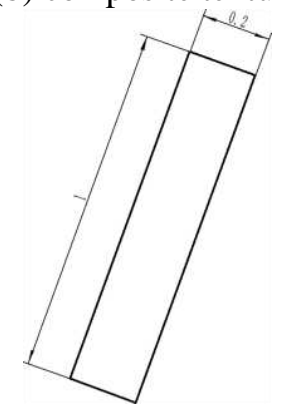

(d) groove texture

Figure 3. The size of the texture

If the thickness of the oil film is too large, it is inconsistent with the reality, and if it is

9 too small, the hydrodynamic lubrication effect cannot be reflected. The thickness of the oil

10 film was set to half of the texture depth. The meshing adopted a mixed mesh of unstructured

11 tetrahedron and hexahedron. Encryption processing was performed at the boundary of the 
1 surface and near the texture. ICEM CFD was used for meshing. The fluid in the simulation

2 model is lubricating oil. Viscosity grade is ISO VG 68, density is $895 \mathrm{~kg} / \mathrm{m}^{3}$, viscosity is 61.8

$3 \times 10^{-6} \mathrm{~mm}^{2} / \mathrm{s}$. The model selected the k-epsilon Model and used Enhanced Wall Treatment.

4 When setting the input and output boundary conditions of the simulation model, the

5 individual texture model is different from the annular overall texture model. Taking the

6 composite texture as an example, when the texture model is alone, the input face was set as a

7 pressure inlet. The pressure value was calculated according to the loading force values in the

8 actual experiment-50 N, $100 \mathrm{~N}, 150 \mathrm{~N}$ and $200 \mathrm{~N}$. The pressure value was obtained from

9 Equation 9:

$$
P=\frac{F}{S}=\frac{50 \mathrm{~N}}{0.0000887 \mathrm{~m}^{2}}=562429.7 \mathrm{~Pa},
$$

The input pressure is $562429.7 \mathrm{~Pa}$ at a load of $50 \mathrm{~N}$, and was set to $560000 \mathrm{~Pa}$ during simulation. Similarly, the pressure value is $1120000 \mathrm{~Pa}$ at a load of $100 \mathrm{~N}$, is $1680000 \mathrm{~Pa}$ at a load of $150 \mathrm{~N}$, and is $2240000 \mathrm{~Pa}$ at a load of $200 \mathrm{~N}$. The output surface was set as a pressure outlet, and the pressure was the same as inlet. The empirical value of the turbulence intensity was $10 \%$, and the hydraulic diameter was calculated by Equation 10:

$$
D=\frac{4 A}{P}=\frac{4 \cdot 1 \cdot 0.15 \mathrm{~mm}^{2}}{2.3 \mathrm{~mm}}=0.26 \mathrm{~mm}
$$

Where $\mathrm{A}$ is the cross-sectional area of the model and $\mathrm{P}$ is the cross-sectional circumference of the model.

The upper end face was set as the speed inlet. The speed value was based on the speed value in the actual experiment-50 r/min, $100 \mathrm{r} / \mathrm{min}, 120 \mathrm{r} / \mathrm{min}, 150 \mathrm{r} / \mathrm{min}, 180 \mathrm{r} / \mathrm{min}$ and 200 r/min. It can be calculated by Equation 11:

$$
v=\frac{r}{T}=\frac{0.006 \cdot 100 \mathrm{~m} / \mathrm{s}}{60}=0.01 \mathrm{~m} / \mathrm{s}
$$

When the rotation speed is $100 \mathrm{r} / \mathrm{min}$, the average linear velocity of the grinding piece is 0.01 
$1 \mathrm{~m} / \mathrm{s}$. Similarly, when the rotation speeds are $50 \mathrm{r} / \mathrm{min}, 120 \mathrm{r} / \mathrm{min}, 150 \mathrm{r} / \mathrm{min}, 180 \mathrm{r} / \mathrm{min}$ and

$2200 \mathrm{r} / \mathrm{min}$, the corresponding linear velocity values of simulation model are $0.05 \mathrm{~m} / \mathrm{s}, 0.12$

$3 \mathrm{~m} / \mathrm{s}, 0.15 \mathrm{~m} / \mathrm{s}, 0.18 \mathrm{~m} / \mathrm{s}$ and $0.2 \mathrm{~m} / \mathrm{s}$. The algorithm used the SIMPLE method. The

4 momentum equation adopted two-level upwind style discreteness. The remaining energy

5 equations adopted the first-order upwind style discreteness. The relaxation factors remained at

6 their default values. The calculation starting point was the input face.

When the texture model is overall texture. The upper end face was set as the pressure

8 input face, and the pressure value was the same as the single texture model. The inner and

9 outer rings of the model were set as pressure output surfaces. In actual experiments, the

10 pressure on both sides is atmospheric pressure. Therefore, the output surface pressure value in

11 the simulation model is zero. The other surface was set as a moving wall and rotates around

12 the center according to the rotation speed. The algorithm, momentum equation, energy equation and relaxation factor were the same as the individual texture model.

\subsection{Simulation results}

Figure 4 shows the total pressure cloud diagram and dynamic pressure cloud diagram of the four textures under the same experimental conditions $(50 \mathrm{~N}, 100 \mathrm{r} / \mathrm{min})$. As shown in Figure 4(a), the high-pressure areas of the four textures are in the middle of the double-row texture. The high-pressure areas of chevron, composite, and circular textures are more concentrated and the boundaries are obvious, while the high-pressure areas of the groove texture have blurred boundaries, and there is no significant difference in pressure values across the texture surface. From the numerical point of view, the pressure value of the circular texture is the largest, the pressure value of the chevron texture is second only to the circular texture, and the pressure value of the groove texture is the smallest. As shown in Figure 4(b), 
1 the dynamic pressure distribution on the surface of the four textures is basically the same, and

2 they are relatively small. This is because the dynamic pressure value at the position close to

3 the wall is smaller than the center position of the fluid flow.

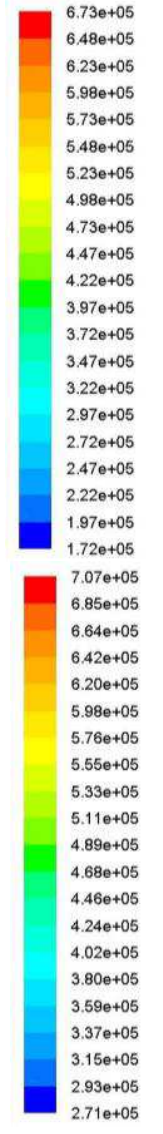

4
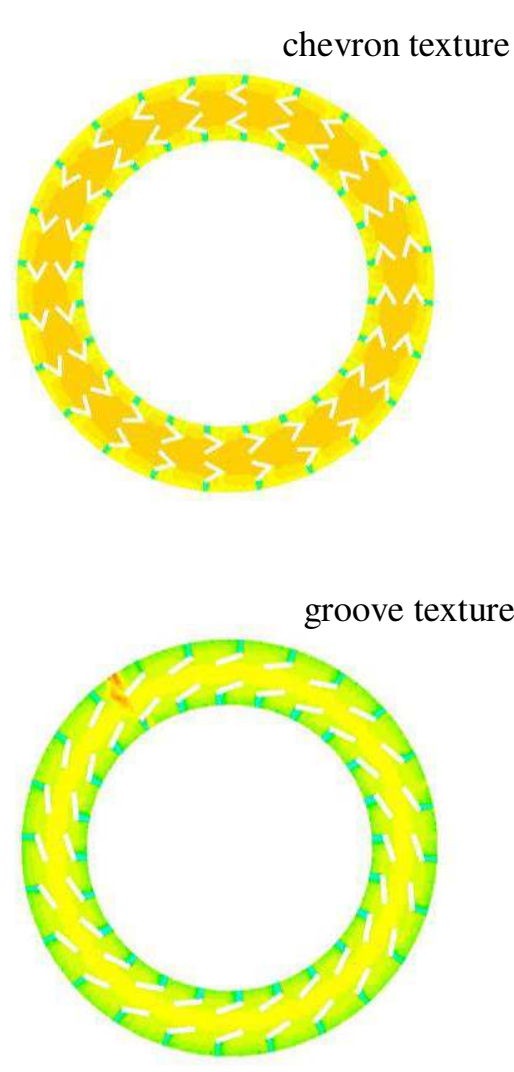

(a) Overall pressure cloud diagram
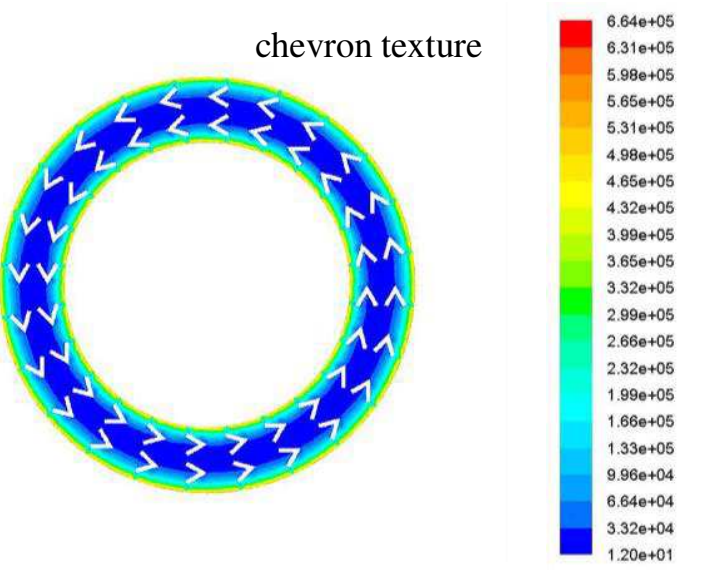
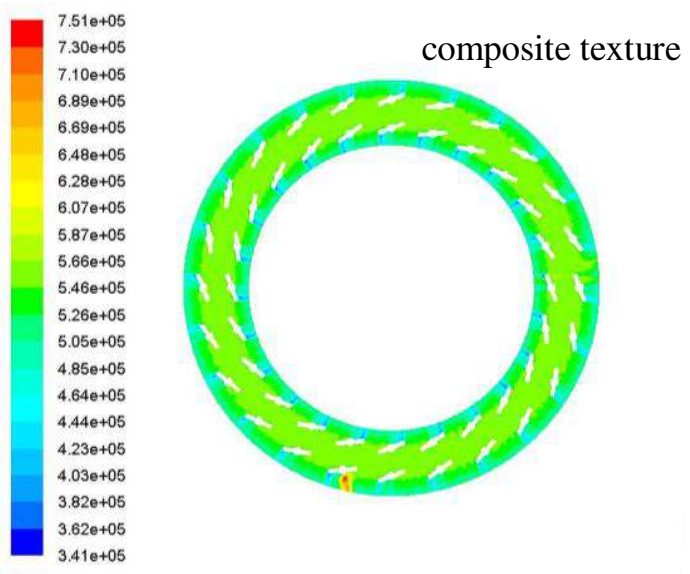

$6.68 e+05$
$6.37 e+05$
$6.06 e+05$
$5.75 e+05$

$5.75 e+05$
$5.44 e+05$

$5.13 \mathrm{e}+05$

$4.82 \mathrm{e}+05$

$4.51 \mathrm{e}+05$

$4.200+05$

$3.890+05$

$3.58 e+05$
$3.27 e+05$

$2.96 e+05$

$2.65 \mathrm{e}+05$

$2.34 \mathrm{e}+05$

$2.03 \mathrm{e}+0$

$1.72 \mathrm{e}+05$
$1.41 \mathrm{e}+05$

$1.10 \mathrm{e}+0$

$7910+0$

$7.91 \mathrm{e}+04$
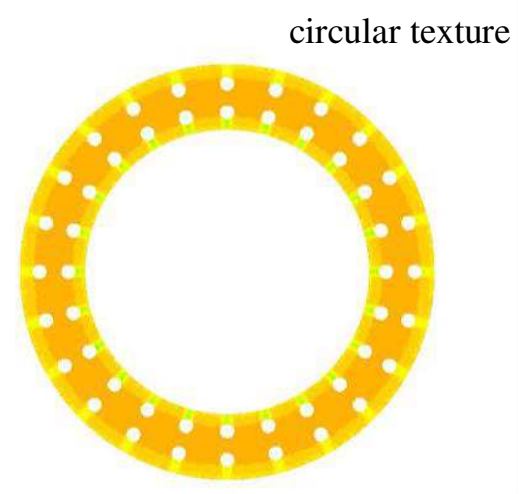

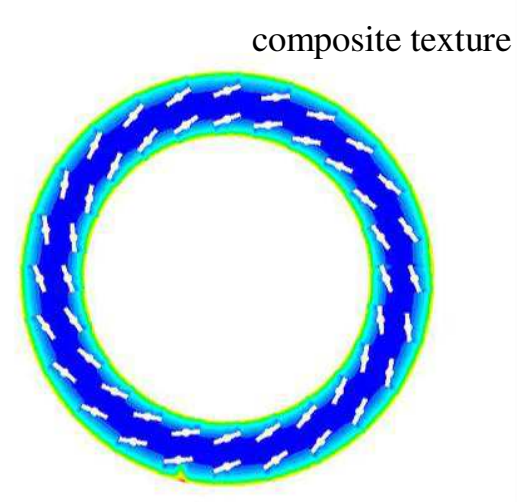




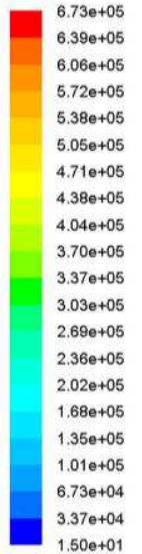

1

2

3

4

5

6

7

8
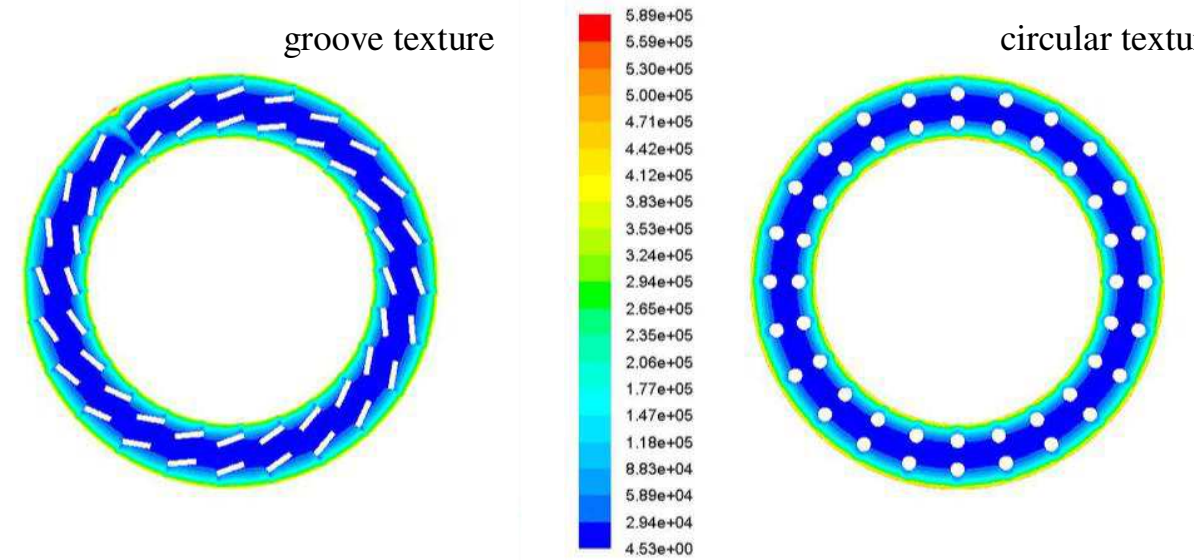

(b) Dynamic pressure cloud diagram

\subsection{Numericalization of simulation results}

\subsubsection{Data collection}

When constructing a mathematical model, the partial derivative of velocity $v$ with respect to $y$ is unknown. Because it is not easy to measure in actual experiments, it needs to be calculated according to the simulation analysis results. Using FLUENT software, the velocity clouds of the four textures are obtained when the loading force is $50 \mathrm{~N}$ and the rotation speed is $100 \mathrm{r} / \mathrm{min}$. The results are shown in Figure 5. According to the simulation results, a vertical line is taken at the output end face, as shown in Figure 6. FLUENT's data processing function is used to output ASCII code data, and then Notepad ++ is used to import the data into an Excel table after processing. The values in the data include $\mathrm{x}$-coordinate, $\mathrm{y}$-coordinate, z-coordinate, and velocity-magnitude. According to the theoretical analysis part, the value of $v_{x}(y)$ is velocity-magnitude. And according to Figure 6, the speed value is $100 \mathrm{~mm} / \mathrm{s}-300$ $\mathrm{mm} / \mathrm{s}$, the actual speed is about $1-10 \mathrm{~mm} / \mathrm{s}$, so in order to rigorous and accurate mathematical model, the data needs to be processed. All simulation values were reduced by 100 times. 

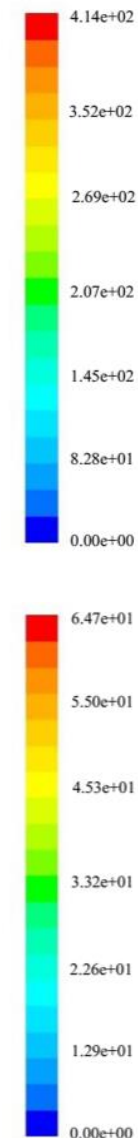

(c) groove texture

(a) chevron texture
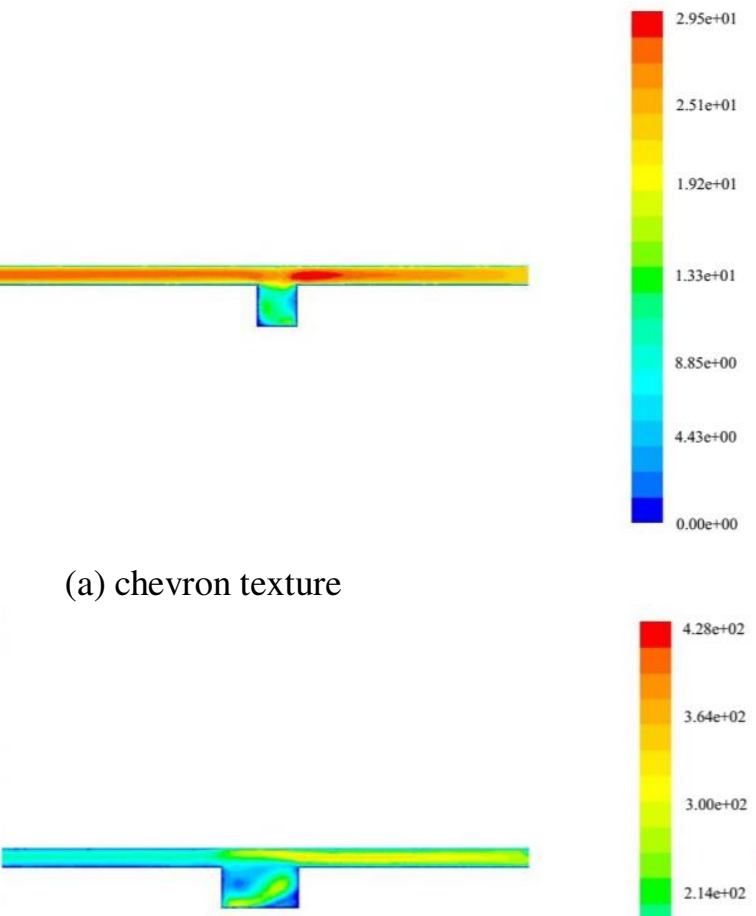

Figure 5. Cloud diagram of velocity distribution

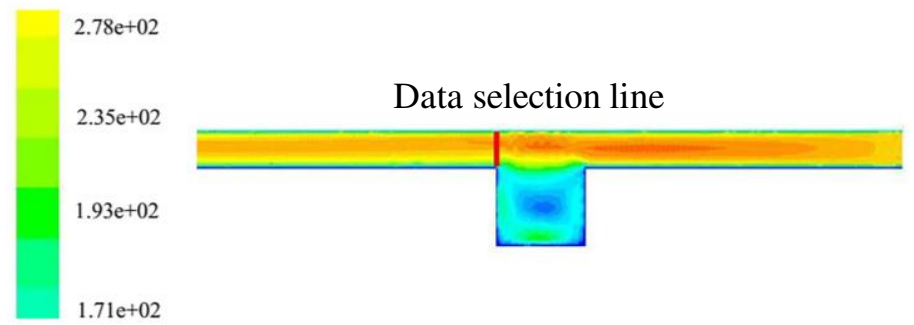

Figure 6. Schematic diagram of the location of the data selection line

\subsubsection{Simulation equations of key variables}

After obtaining the simulation data value, the function equation is solved according to

5 the numerical value, and the solving process is carried out with the help of MATLAB toolbox.

6 The processed data was imported into the MATLAB workspace and named for different data

7 groups. The Curve Fitting toolbox was selected to perform curve fit analysis on the data in the

8 work area to obtain a function equation. Figure 7 shows the fitting curves of four textures.

9 The abscissa is the $y$ value, and the y-axis zero position is at the top of the data selection line 
1 in Figure 6. The ordinate is the speed value. R-square is the degree of equation fit. To

2 facilitate the solution of the mathematical model, replace $y$ in the original formula with $x$. The

3 four texture fitting equations are shown in Table 1.

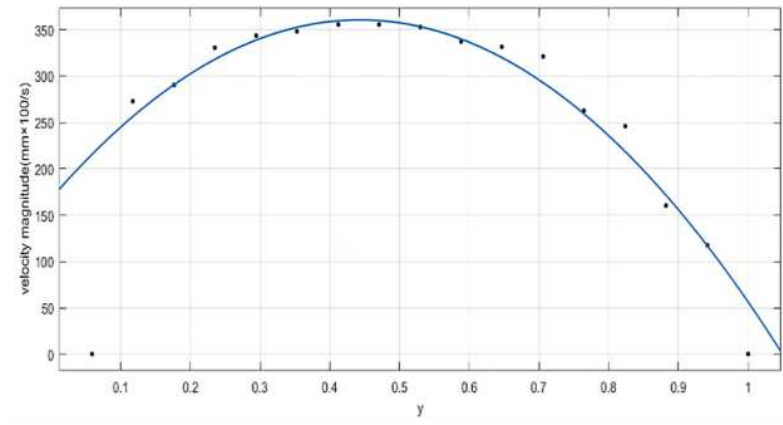

(a) chevron texture

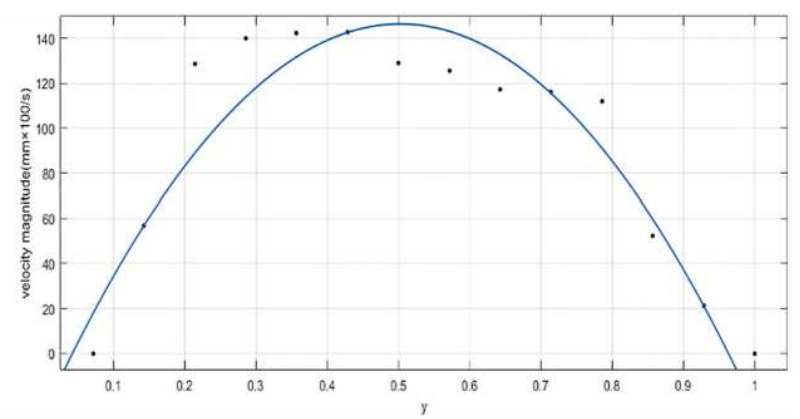

(c) groove texture

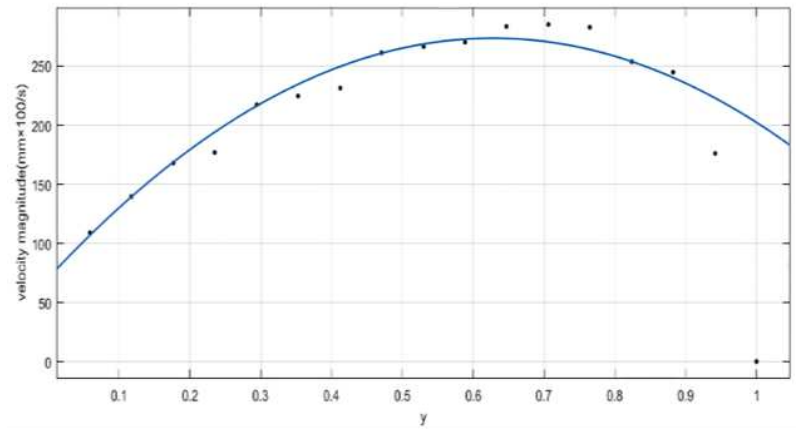

(b) composite texture

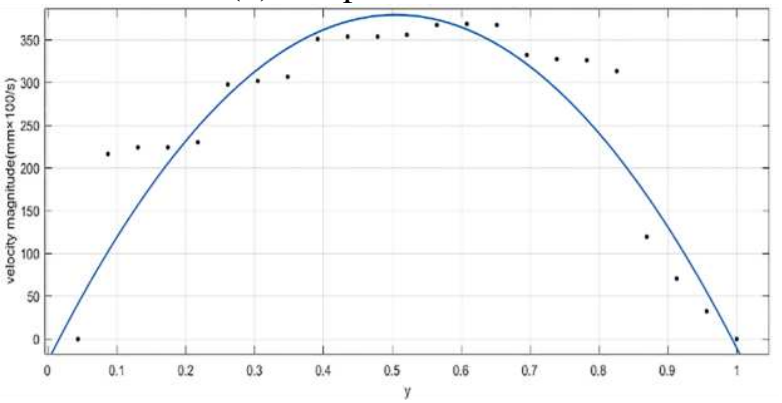

(d) circular texture

Figure 7. Fitting curves of four textures

Table 1. Simulation equations of four textures

\begin{tabular}{ccccc}
\hline Texture & Chevron texture & Composite texture & Groove texture & Circular texture \\
\hline Equation & $f(x)=P_{1} \cdot x^{2}+P_{2} \cdot x+$ & $f(x)=P_{1} \cdot x^{2}+P_{2} \cdot x+$ & $f(x)=P_{1} \cdot x^{2}+P_{2} \cdot x+$ & $f(x)=P_{1} \cdot x^{2}+P_{2} \cdot x+$ \\
& $P_{3}$ & $P_{3}$ & $P_{3}$ & $P_{3}$ \\
$P_{1}$ & -9.52 & -5.146 & -6.904 & -15.90 \\
$P_{2}$ & 8.377 & 6.462 & 6.942 & 16.06 \\
$P_{3}$ & 1.791 & 0.7071 & -0.2811 & -0.2623 \\
R-square & 0.946 & 0.912 & 0.8769 & 0.8741 \\
\hline
\end{tabular}

\section{Friction and wear experiment}

\section{$7 \quad 4.1$ Design of the experiment}

\subsubsection{Design of surface texture}

The area occupancy rate of the four textures is $10 \%$. The processing parameters of the 
1 four textures are shown in Table 2.

2

Table 2. Processing parameters of four textures

\begin{tabular}{cc|c|c|c}
\hline Texture & Chevron texture & Composite texture & Groove texture & Circular texture \\
\hline Distribution form & Double row & Double row & Double row & Double row \\
Unit number & 48 & 48 & 48 & 48 \\
Area of unit texture & $0.2 \mathrm{~mm}^{2}$ & $0.19 \mathrm{~mm}^{2}$ & $0.2 \mathrm{~mm}^{2}$ & $0.2 \mathrm{~mm}^{2}$ \\
Area occupancy rate & $10.8 \%$ & $10 \%$ & $10.8 \%$ & $10.8 \%$ \\
\hline
\end{tabular}

Before processing the texture on the surface of the workpiece, it is necessary to pre-treat

4 the test piece. Boron carbide powder was used to grind the test piece to smooth on frosted

5 glass. Diamond polishing paste was used to polish the test piece on the polishing machine

6 until the surface was mirror-like. The test piece was placed in an ultrasonic cleaner to clean

7 the surface dirt. The polished samples were measured on a Mitutoyo SJ-410 surface

8 roughness measuring instrument and the average surface roughness of all test pieces was

$9 \quad$ 0.045. A Libra-HE femtosecond laser was used to process the surface texture.
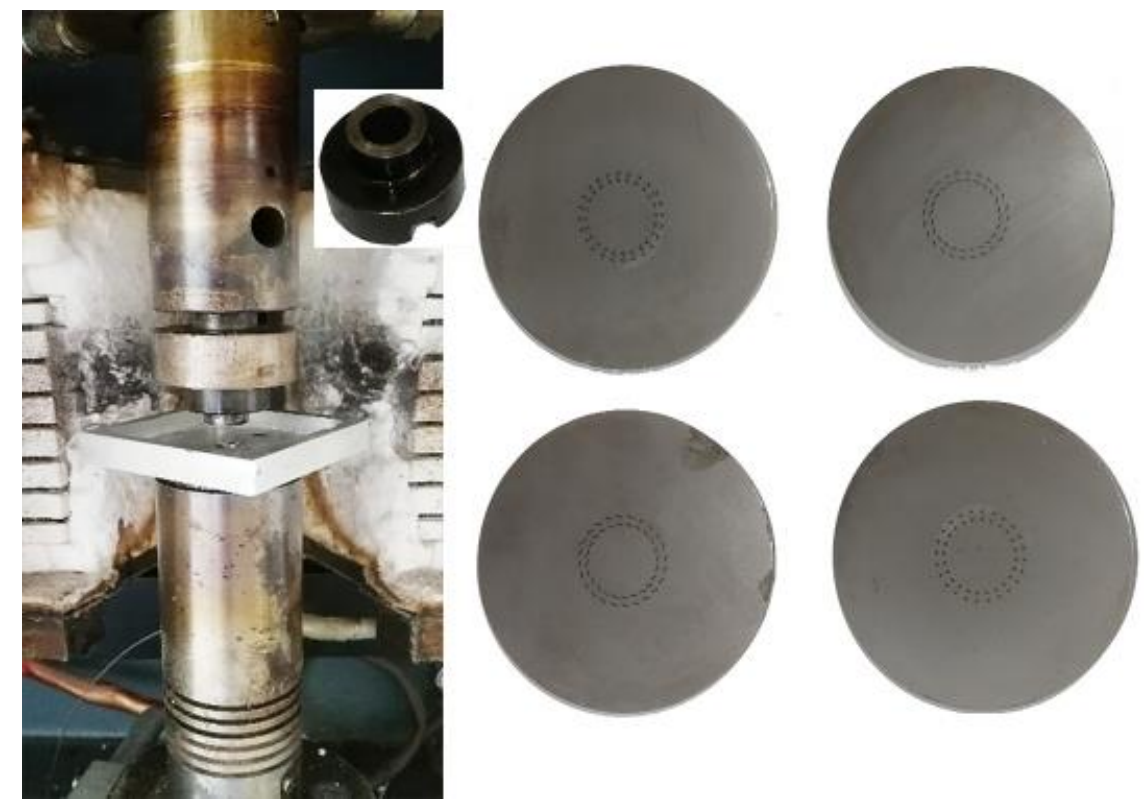

Figure 8. Friction and wear testing machine, 45 steel, and workpiece after processing texture

\subsubsection{Experimental scheme}

The experiment used MMG-10 friction and wear testing machine. The lubricating oil 
1 loading force and speed, and the parameter values are shown in Table 3. Four variables were

2 selected for the loading force and six variables for the rotation speed, and a total of 96 groups

3 of experiments were conducted, each group of experiments for 10 minutes.

Table 3. Experimental parameter values

\begin{tabular}{ccccc}
\hline Texture & $\begin{array}{c}\text { Loading } \\
\text { force }(\mathrm{N})\end{array}$ & $\begin{array}{c}\text { Rotation } \\
\text { speed }(\mathrm{r} / \mathrm{min})\end{array}$ & $\begin{array}{c}\text { Condition } \\
\text { Contact } \\
\text { method }\end{array}$ & \\
Chevron texture & 50 & 100 & $25^{\circ} \mathrm{C}$ & \\
Composite texture & 100 & 120 & Atmospheric pressure & Face to face \\
Groove texture & 150 & 150 & Oil lubrication & \\
Circular texture & 200 & 180 & & \\
& & 200 & & \\
\hline
\end{tabular}

\section{$5 \quad 4.2$ Experimental results}

\section{$6 \quad$ 4.2.1 Chevron texture}
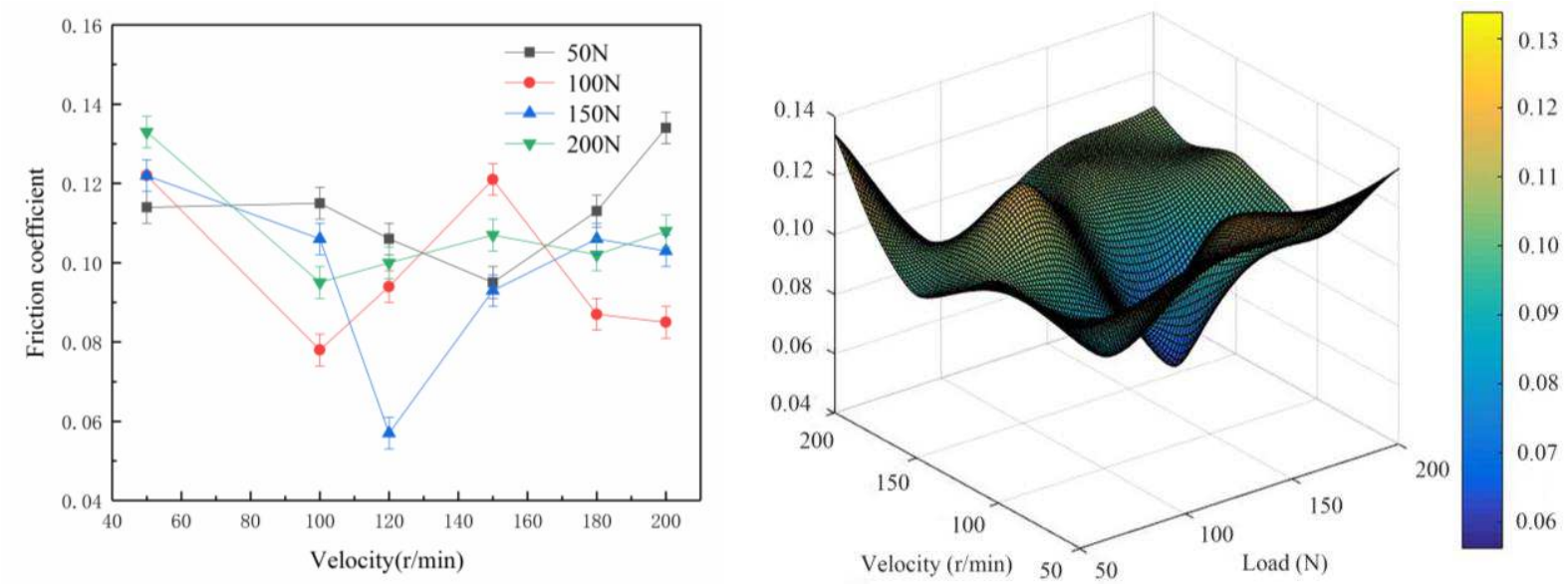

Figure 9. Experimental results of chevron texture

Figure 9 is the experimental results of chevron texture under different loading force and speed. According to the three-dimensional graph, the distribution interval of the friction coefficient of chevron texture is $[0.05,0.14]$, and the range is 0.09 . The lowest point of the entire curved surface is when the loading force is $150 \mathrm{~N}$ and the rotation speed is $120 \mathrm{r} / \mathrm{min}$. The highest points are at the loading force of $200 \mathrm{~N}$ and the speed of $50 \mathrm{r} / \mathrm{min}$ and the loading force of $50 \mathrm{~N}$ and the speed of $200 \mathrm{r} / \mathrm{min}$, respectively. The point where the friction coefficient 
1 is the smallest is the value when the loading force and the rotation speed are at an

2 intermediate level. The friction coefficient at a loading force of $50 \mathrm{~N}$ and a rotation speed of

$350 \mathrm{r} / \mathrm{min}$ and a loading force of $200 \mathrm{~N}$ and a rotation speed of $200 \mathrm{r} / \mathrm{min}$ is close and small.

4 This shows that there is an optimal matching interval between loading force and speed. When

5 the difference between loading force and speed is too large, the friction coefficient will

6 increase. When the speed is constant, the friction coefficient decreases with increasing loading

7 force. When the loading force is constant, the friction coefficient decreases with increasing

8 speed. When the loading force is $200 \mathrm{~N}$, the friction coefficient is small and the change is

9 relatively stable, indicating that the chevron texture shows good hydrodynamic lubrication

10 characteristics under high load conditions. It proves that the chevron texture has strong

11 pressure bearing capacity and is suitable for high load conditions.

\subsubsection{Composite texture}
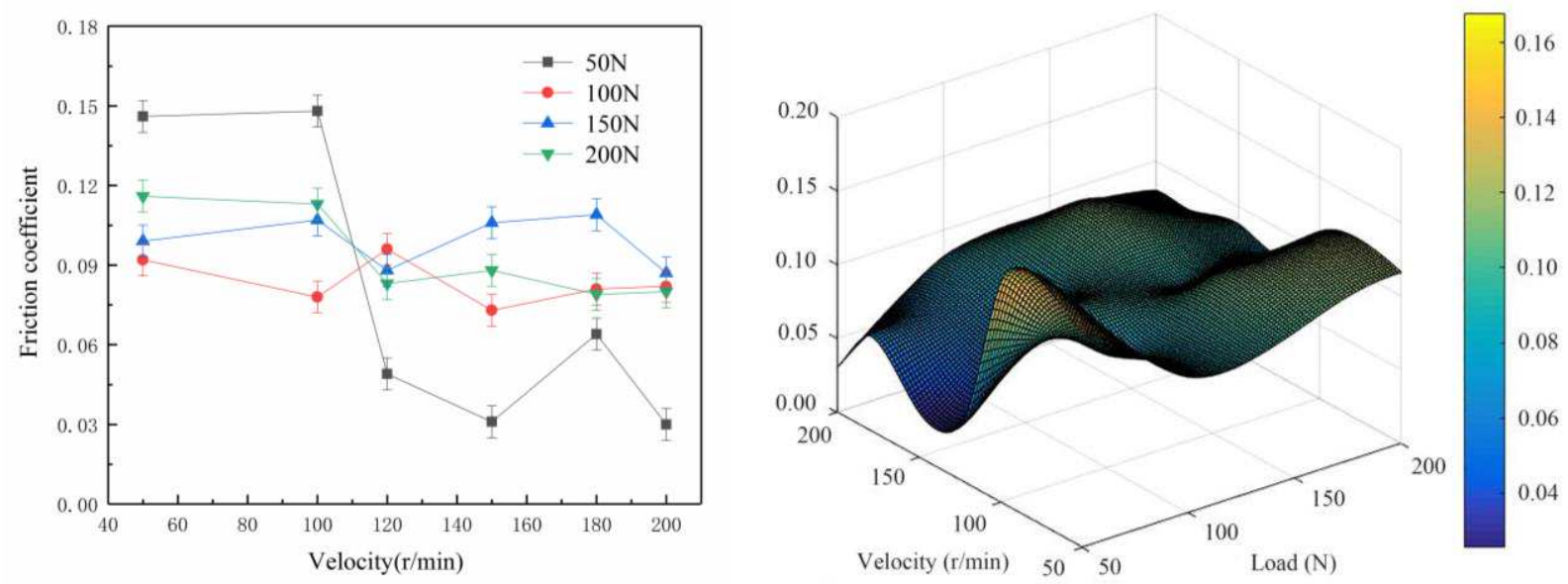

Figure 10. Experimental results of composite texture

According to the three-dimensional diagram of Figure 10, the distribution interval of the

friction coefficient of the composite texture is [0.03, 0.148], and the range is 0.118 . The entire

curved surface presents a two-step shape. The lowest point of the curved surface is when the 
1 rotation speed is $50 \mathrm{r} / \mathrm{min}$, and the loading force is $50 \mathrm{~N}$ and the rotation speed is $100 \mathrm{r} / \mathrm{min}$.

2 Both the maximum and minimum friction coefficients are when the loading force is $50 \mathrm{~N}$.

3 When the rotation speed is greater than $100 \mathrm{r} / \mathrm{min}$, the friction coefficient will appear a

4 stepwise decline under each loading force, and then it will tend to be gentle. When the loading

5 force is $50 \mathrm{~N}$, the range of friction coefficient is extremely large, and the fluctuation is severe.

6 At low speeds, the friction coefficient increases with increasing loading force. At high speeds,

7 the friction coefficient decreases with decreasing loading force. This shows that for composite

8 textures, the influence of speed is greater than the loading force. As shown in the line chart,

9 when the rotation speed is constant, the friction coefficient fluctuates with the increase of the

10 loading force. When the loading force is constant, the friction coefficient decreases with

11 increasing speed. When the loading force is $100 \mathrm{~N}$ and above, the friction coefficient value is

12 small and tends to be stable. When the loading force is $100 \mathrm{~N}$, the friction coefficient is more

13 stable and smaller than when the loading force is $150 \mathrm{~N}$ and $200 \mathrm{~N}$. It shows that the

14 composite texture shows good hydrodynamic lubrication characteristics under high-speed and

15 medium-high load conditions. It is proved that the composite texture contains the

16 characteristics of groove texture and circular texture, and has a certain pressure-bearing

17 capacity and the ability to promote the flow of lubricating oil.

$18 \quad$ 4.2.3 Groove texture 

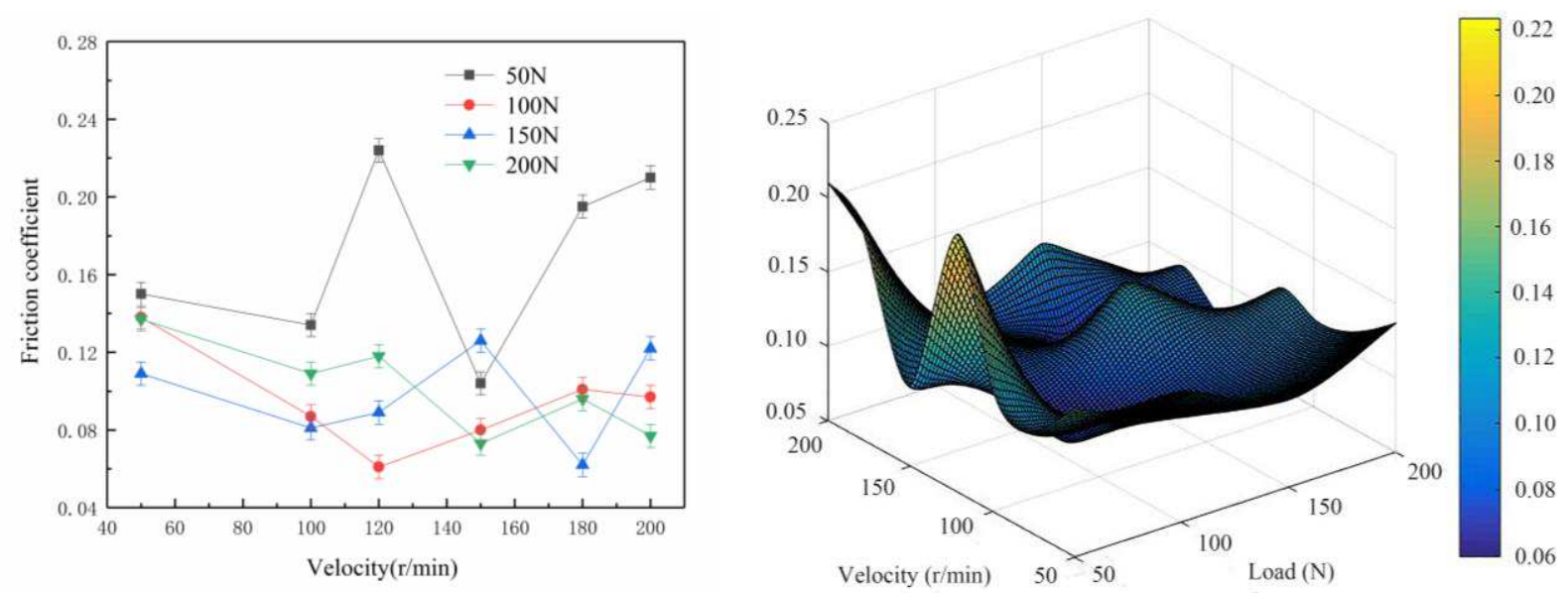

Figure 11. Experimental results of groove texture

According to the three-dimensional graph of Figure 11, the distribution interval of the groove-type texture friction coefficient is $[0.061,0.224]$, and the range is 0.163 . The overall figure presents a double hump-like parallel distribution. The highest point is when the loading force is $50 \mathrm{~N}$ and the speed is $120 \mathrm{r} / \mathrm{min}$. The lowest points are when the loading force is 100 $\mathrm{N}$ and the rotation speed is $120 \mathrm{r} / \mathrm{min}$, and the loading force is $150 \mathrm{~N}$ and the rotation speed is $180 \mathrm{r} / \mathrm{min}$. When the loading force is $50 \mathrm{~N}$, the friction coefficient fluctuates violently and the range is large. When the loading force is $200 \mathrm{~N}$, the surface changes smoothly and the friction coefficient is stable. When the loading force is constant, the friction coefficient is distributed in a hump shape as the speed increases. When the speed is constant, the friction coefficient is distributed in a hump shape as the loading force increases. As shown in the line chart, there is no obvious stepwise decline and level change in the friction coefficient when the loading force is $100 \mathrm{~N}, 150 \mathrm{~N}$ and $200 \mathrm{~N}$. When the rotation speed is between $100-150 \mathrm{r} / \mathrm{min}$, each loading force has a small friction coefficient. It shows that the groove texture shows good anti-friction characteristics under medium speed conditions. It is proved that the groove texture has weak pressure bearing capacity and is suitable for medium speed and low load conditions. 

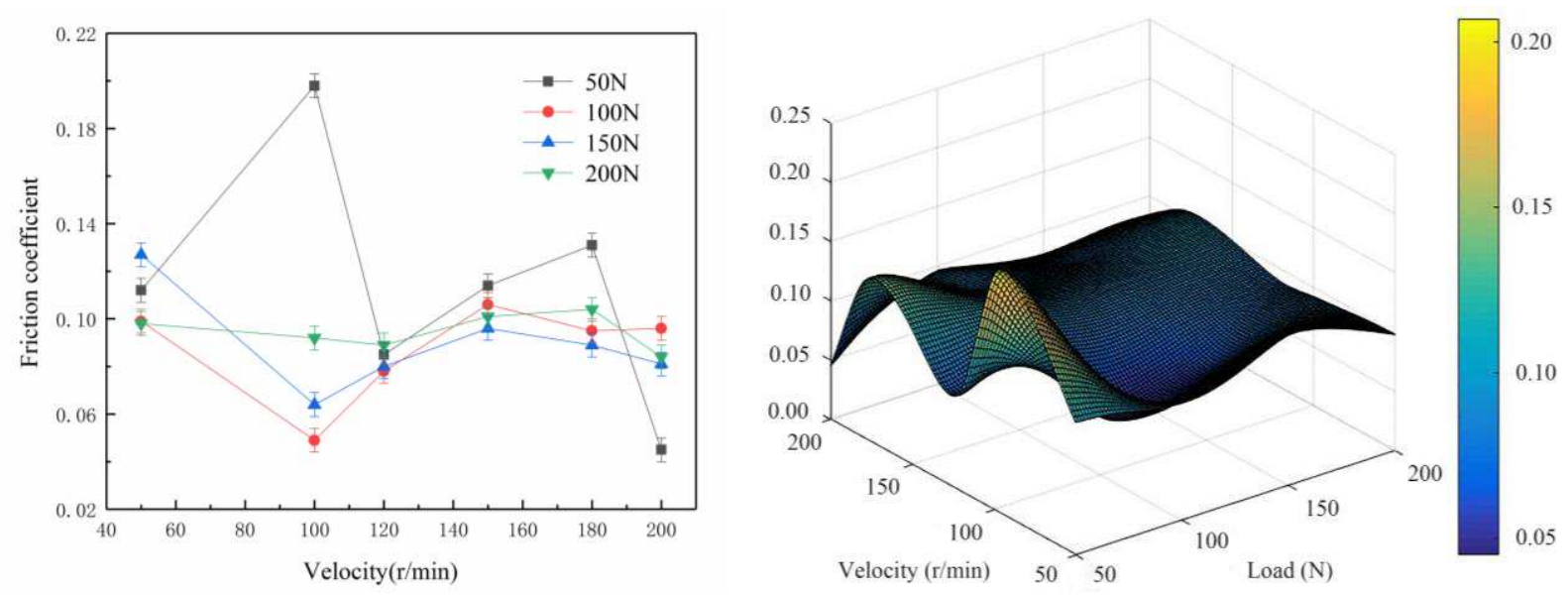

Figure 12. Experimental results of circular texture

(1)

According to the three-dimensional diagram of Figure 12, the distribution interval of the circular texture friction coefficient is $[0.045,0.198]$, and the range is 0.153 . The highest point is when the loading force is $50 \mathrm{~N}$ and the speed is $100 \mathrm{r} / \mathrm{min}$. The lowest points are when the loading force is $100 \mathrm{~N}$ and the rotation speed is $100 \mathrm{r} / \mathrm{min}$, and the loading force is $50 \mathrm{~N}$ and the rotation speed is $200 \mathrm{r} / \mathrm{min}$. The friction coefficient fluctuates greatly when the loading force is $50 \mathrm{~N}$, and the curved surface is hump-shaped. The friction coefficient gradually stabilizes with the increase of the loading force, and it shows a plane-like distribution. As shown in the line chart, when the speed is constant, the friction coefficient decreases with the increase of the loading force. When the loading force is $200 \mathrm{~N}$, the friction coefficient is small and the change is relatively stable. When the loading force is constant, the friction coefficient increases with the increase of speed. When the rotation speed is $100 \mathrm{r} / \mathrm{min}$ and the loading force is $100 \mathrm{~N}, 150 \mathrm{~N}, 200 \mathrm{~N}$, the friction coefficient is the minimum value under the loading force. It shows that the circular texture shows excellent stability under high load and medium speed conditions. It is proved that the circular texture has strong pressure bearing capacity and strong stability, and is suitable for high load conditions.

\subsubsection{Friction coefficients of different textures under the same loading force}




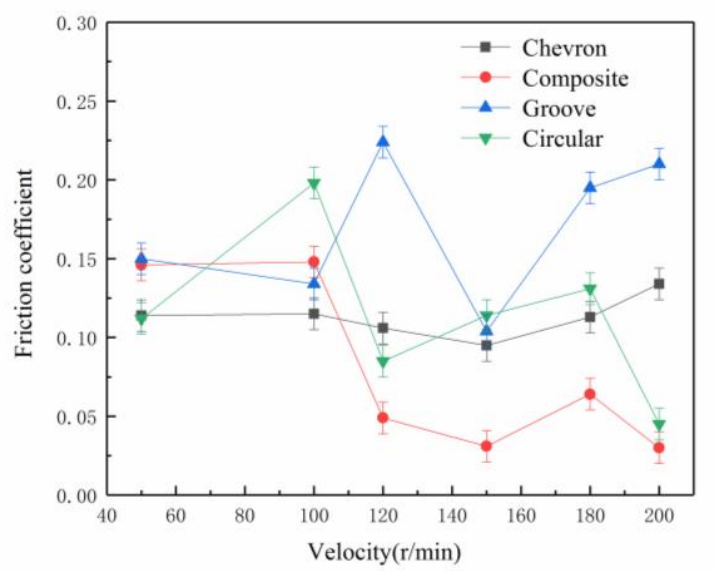

(a) $50 \mathrm{~N}$

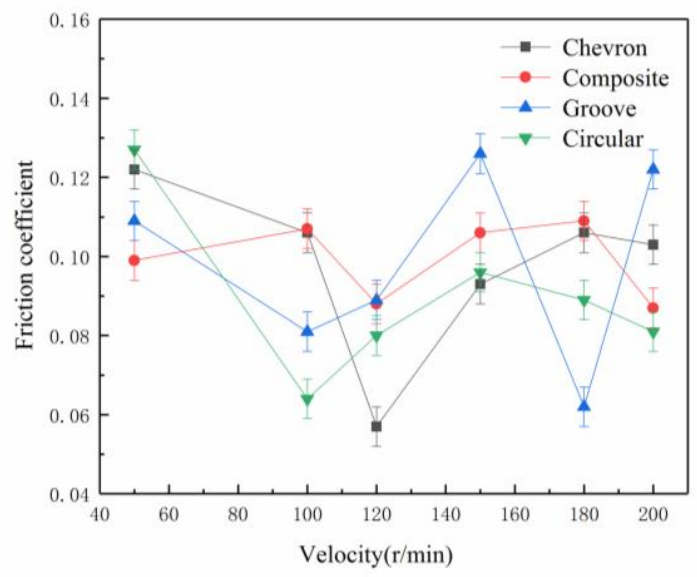

(c) $150 \mathrm{~N}$

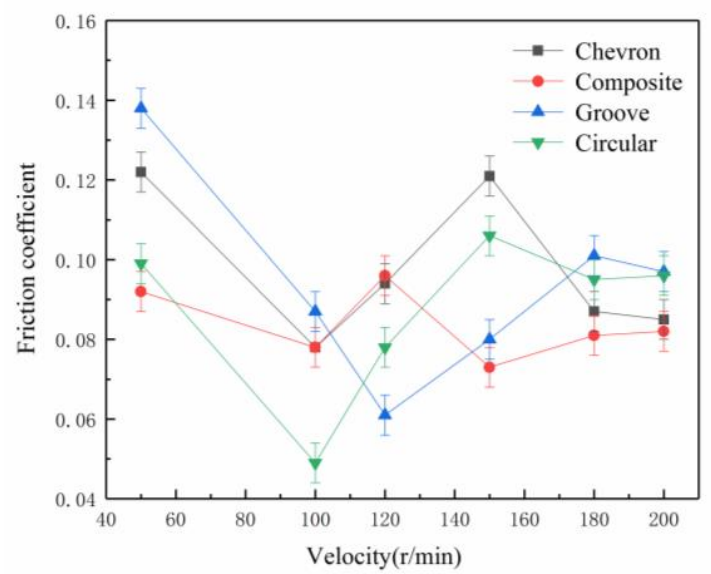

(b) $100 \mathrm{~N}$

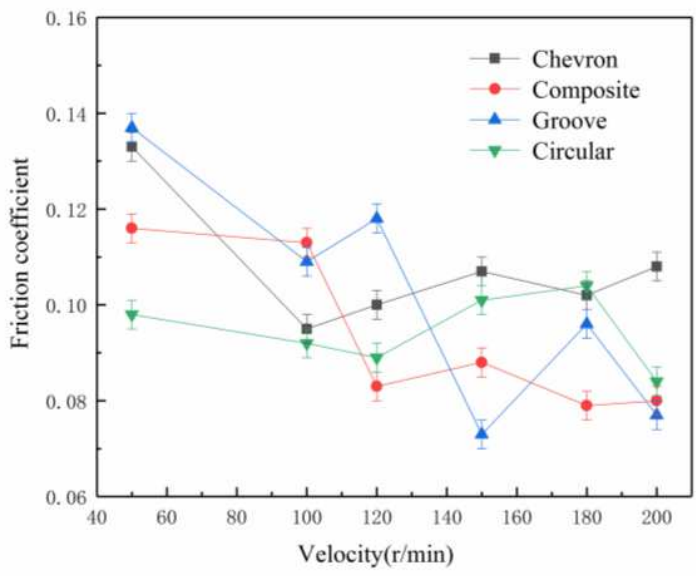

(d) $200 \mathrm{~N}$
4 friction coefficient of chevron texture and circular texture is the lowest. At high speeds, the

5 friction reduction effect of the composite texture is most obvious. When the loading force is

$6 \quad 150 \mathrm{~N}$, the friction coefficient of chevron, composite and circular texture changes in the same 8 coefficient of the circular texture is $100 \mathrm{r} / \mathrm{min}$, the chevron texture is $120 \mathrm{r} / \mathrm{min}$, and the 9 groove texture is $180 \mathrm{r} / \mathrm{min}$. The friction coefficient of the composite texture changes steadily and there are no huge fluctuations. When the loading force is $200 \mathrm{~N}$ and the rotation speed is

Figure 13. Variation curve of friction coefficient of different textures under the same loading force

Figure 13 is the variation curve of friction coefficient of different textures under the same loading force. When the loading force is $50 \mathrm{~N}, 100 \mathrm{~N}$ and the rotation speed is low, the trend, showing a large undulating wave shape. The rotation speed at the lowest friction and there are no huge fluctuations. When the loading force is $200 \mathrm{~N}$ and the rotation speed is low, the friction coefficient of chevron texture and circular texture is lower. At high speeds, 
1 the friction coefficient of the composite texture and the groove texture is lower. The chevron

2 texture has a strong pressure-bearing ability, but it can only play a good anti-friction effect at

3 low speeds, and the anti-friction effect decreases significantly at high speeds. Due to the weak

4 pressure bearing capacity of the groove texture, the friction coefficient is unstable under high

5 load. However, due to the characteristics of improving fluidity, the friction coefficient is small

6 at high speeds. The composite texture consists of groove and circle. The composite texture

7 still shows good anti-friction effect under high load and high speed working conditions.

8 The above research shows that the chevron texture has a small and stable friction

9 coefficient at high loads. It shows that chevron texture is suitable for high load conditions.

10 The composite texture has a small friction coefficient under high-speed and medium-high

11 load conditions. It shows that the composite texture is suitable for high-speed and

12 medium-high load conditions. The friction coefficient of the groove texture does not decrease significantly when the loading force increases. When the rotation speed is constant, the

14 friction coefficient decreases as the loading force decreases. It shows that the pressure capacity of the groove texture is weak, and it is suitable for medium speed and low load working conditions. The friction coefficient of the circular texture tends to be stable as the

17 loading force increases. When the loading force is constant, the friction coefficient will increase with the increase of speed. It shows that the circular texture has strong pressure bearing capacity and weak flow promotion ability, which is suitable for medium and low speed and high load working conditions.

\section{Solution and verification of mathematical model}

\subsection{Combination of simulation formula and theoretical formula}

\subsubsection{Determination of key parameters}


1 According to the simulation model, the oil flow velocity equations under different

2 surface texture conditions are obtained, and the first derivative is obtained by combining the

3 mathematical model with the results. The results are shown in Table 4. After obtaining the key

4 equation, it is substituted into the theory equation.

5

Table 4. Derivatives of four texture equations

\begin{tabular}{ccccc}
\hline Texture & Chevron texture & Composite texture & Groove texture & Circular texture \\
\hline \multirow{2}{*}{ Equation } & $F^{\prime}(x)=2^{*} K_{1} *^{*} x+$ & $F^{\prime}(x)=2 \cdot K_{1} \cdot x+$ & $F^{\prime}(x)=2 \cdot K_{1} \cdot x+$ & $F^{\prime}(x)=2 \cdot K_{1} \cdot x+$ \\
& $K_{2}$ & $K_{2}$ & $K_{2}$ & $K_{2}$ \\
$K_{1}$ & -9.52 & -6.904 & -6.904 & -15.90 \\
$K_{2}$ & 8.377 & 6.942 & 6.942 & 16.06 \\
\hline
\end{tabular}

6

7

8

\subsubsection{Substitution of key parameters}

The above key equation is substituted into Equation 8 to obtain Equation 12:

$u=\frac{P \cdot(1-x) \cdot 0.002124}{0.0083 \cdot\left(U-F^{\prime}(x)\right)}$

Equation 12 is further simplified to obtain Equation 13:

$$
u=\frac{0.256 \cdot P(1-x)}{\left(U-2 \cdot K_{1} \cdot x-K_{2}\right)}
$$

Where $K_{1}$ and $K_{2}$ are equation parameter values of different surface textures. The equations of the four surface textures are as follows.

The equation of chevron texture is:

$$
u=\frac{0.256 \cdot P(1-x)}{(U+19.04 \cdot x-8.377)}
$$

5 The equation of composite texture is:

16

$$
u=\frac{0.256 \cdot P(1-x)}{(U+10.292 \cdot x-6.462)}
$$


1 The equation of groove texture is:

$2 \quad u=\frac{0.256 \cdot P(1-x)}{(U+13.808 \cdot x-6.942)}$

3 The equation of circular texture is:

$4 \quad u=\frac{0.256 \cdot P(1-x)}{(U+31.80 \cdot x-16.06)}$

\section{$5 \quad 5.2$ Verification of formula}

6

7 load $P$, and the rotation speed $U$ can be obtained, and the value range of $x$ is $[0,1]$. After

8 substituting the loading force and speed under different working conditions into equations 14 ,

915,16 , and 17 , the minimum value of the equation in the interval $[0,1]$ is solved, and the

According to the above equation, the relationship between the friction coefficient $u$, the function values of the four equations are compared. The texture with a smaller function value is the better texture, which can achieve the purpose of selecting the optimal texture according to different working conditions.

In order to verify the accuracy of the formula, an experimental working condition was selected to compare the experimental results with the calculation results of the formula. The loading force of $50 \mathrm{~N}$ and the speed of $100 \mathrm{r} / \mathrm{min}$ are selected to solve the minimum value of the equation. MATLAB software is used to solve the other program. When substituting into the equation, $P$ needs to adopt a unified international unit system, $P=560000 \mathrm{~Pa}$. The calculation results and experimental results are shown in Table 5.

Table 5. Calculation results of MATLAB

\begin{tabular}{ccc}
\hline Texture & Calculation results & Experimental results \\
\hline Chevron texture & 0.0856 & 0.115 \\
Composite texture & 0.0913 & 0.148 \\
Groove texture & 0.0887 & 0.134 \\
& 25 &
\end{tabular}


1

2

According to the calculation results, the order of anti-friction performance of different textures from good to bad is as follows: circular, chevron, groove, composite. According to the experimental results, the order of anti-friction performance of different textures from good to bad is as follows: chevron, groove, composite, circular. In terms of friction coefficient, there is a $10 \%-40 \%$ error between the theoretical analysis results and the experimental results.

From the simulation analysis, it can be seen that the circular texture is suitable for medium and low speed and high load conditions, and $50 \mathrm{~N}, 100 \mathrm{r} / \mathrm{min}$ are medium speed and low load conditions. The simulation results are consistent with MATLAB calculation results. An important factor that may cause large differences in experimental results may be experimental errors. According to the friction and wear test results of circular texture, the friction coefficient is large under this condition, indicating that there is a greater possibility of errors. Other working conditions were followed the above steps to verify the hydrodynamic lubrication theory. The results prove that the theory has a certain accuracy, but it still has some differences from the actual experimental results, and in-depth research is still needed.

\section{Conclusions}

In this paper, taking the severe working condition of the friction surface of the key workpiece as the application background, the mathematical model of hydrodynamic lubrication was constructed through theoretical analysis and simulation. The mathematical model was verified through the friction and wear experiment. The purpose is to summarize a set of surface texture theory and optimal selection equations for complex working conditions.

It is found that under the same area occupancy rate, texture quantity and experimental conditions, different textures have different tribological characteristics under different 
1 working conditions. Circular texture has the strongest pressure-bearing capacity, but the

2 correlation between texture units is weak. The hydrodynamic lubrication effect is not

3 significant at high speeds, and it is suitable for medium and low speed high load conditions.

4 The bearing capacity of chevron texture is second only to circular texture, and the correlation

5 between texture units is better than circular texture, which is suitable for medium and high

6 speed, medium and high load conditions. The pressure capacity of the groove texture is the

7 weakest among the four textures, but the correlation of the texture unit is the best, which is

8 more suitable for high speed and low load working conditions. The composite texture

9 includes the characteristics of circular texture and groove texture. It has certain pressure

10 bearing capacity and good correlation of texture units. It is suitable for high-speed and

11 medium-high load conditions. The mathematical model is verified by the experimental results,

12 and the results show the accuracy and reliability of the theory.

13 Funding:

14 The National Natural Science Foundation of China (51575234, 51872122) and the 15 Postdoctoral Science Foundation of China (2017M620286) and The Key Research and 16 Development Program of Shandong Province, China (2018CXGC0809) and Agricultural 17 Machinery Equipment Research and Development Innovation Plan of Shandong Province 18 (2018YF012), Experts from Taishan Scholars and Youth Innovation in Science \& Technology Support Plan of Shandong Province University.

\section{References}

[1] Y.Q. Xing, J.X. Deng, Z. Wu, High friction and low wear properties of laser-textured ceramic surface under dry friction, OPT. LASER. TECHNOL. 93 (2017) 24-32.

[2] D. Bhaduri, A. Batal, S.S. Dimov, On Design and Tribological Behaviour of Laser Textured Surfaces, Procedia CIRP. 60 (2017) 20-25. 
1 [3] H.Z. Fan, Y.F. Su, J.J. Song, Design of "double layer" texture to obtain superhydrophobic and high wear-resistant PTFE coatings on the surface of $\mathrm{Al}_{2} \mathrm{O}_{3} / \mathrm{Ni}$ layered ceramics, TRIBOL. INT. 136 (2019) 455-461.

[4] Y.K. Zhou, H. Zhu, W. Tang, Development of the theoretical model for the optimal design of surface texturing on cylinder liner, TRIBOL. INT. 52 (2012) 1-6.

[5] Z. Zhao, W.Z. Lu, Y.F. He, Research on optimal laser texture parameters about antifriction characteristics of cemented carbide surface, INT. J. REFRACT. MET. H. 82 (2019) 287-296.

[6] G. Ryk, I. Etsion, Testing piston rings with partial laser surface texturing for friction reduction, WEAR. 261 (2006) 792-796.

[7] I. Etsion, Y. Kligerman, G. Halperin, Analytical and experimental investigation of laser-textured mechanical seal faces, A S L E Transactions. 42 (1999) 511-516.

[8] M. Wakuda, Y. Yamauchi, S. Kanzaki, Effect of surface texturing on friction reduction between ceramic and steel materials under lubricated sliding contact, WEAR. 254 (2003) $356-363$.

[9] K. TONDER, Hydrodynamic effects of tailored inlet roughnesses: Extended theory, TRIBOL. INT. 37 (2004) 137-142.

[10] M. Fowell, A.V. Olver, A.D. Gosman, Entrainment and inlet suction: Two mechanisms of hydrodynamic lubrication in textured bearings, J. TRIBOL-T. ASME. 129 (2007) $336-347$.

[11] A. Hoppermann, M. Kordt, Tribological optimization using laser structured contact surfaces, O+P Oethydraulik and Pneumatik. 46 (2002) 385-392.

[12]I. Křupka, M. Vrbka, M. Vaverka, Effect of surface dents on contact pressure in elastohydro dynamic contacts, P. I. MECH ENG. J-J. ENG. 223 (2009) 683-693.

[13]I. Křupka, M. Vrbka, M. Hartl, Effect of surface texturing on mixed lubricated nonconformal contacts, TRIBOL. INT. 41 (2008) 1063-1073. 
1 [14]R. Rahmani, I. Mirzaee, A. Sshirvani, An analytical approach for analysis and optimization of slider bearings with infinite width, TRIBOL. INT. 43 (2010) 1551-1565.

[15] A.G. Charitopoulos, E.E. Efstathiou, C.I. Papadopoulos, Effects of manufacturing errors on tribological characteristics of 3D textured micro-thrust bearings, CIRP. J. MANUF. SCI. TEC. 6 (2013) 128-142.

[16]P. Andersson, J. Koskinen, S. Varjus, Microlubrication effect by laser-textured steel surfaces, WEAR. 262 (2007) 369-379.

[17] Y. Kligerman, I. Etsion, Analysis of the hydrodynamic effects in a surface textured circumferential gas seal, TRIBOL. T. 44 (2001) 472-478.

[18] A.Y. Suh, S.C. Lee, A. Polycarpou, Adhesion and friction evaluation of textured slider surfaces in ultra-low head-disk interface, TRIBOL. LETT. 17 (2004) 739-749.

[19]R.T. Tong, B. Han, Z.F. Quan, Molecular dynamics simulation of friction and heat properties of Nano-texture GOLD film in space environment, SURF. COAT .TECH. 358 (2019) 775-784.

[20]Z.Y. Li, W.J. Yang, Y.P. Wu, Role of humidity in reducing the friction of graphene layers on textured surfaces, APPL. SURF. SCI. 403 (2017) 362-370.

[21]X.Q. Hao, W. Cui, L. Li, Cutting performance of textured polycrystalline diamond tools with composite lyophilic/lyophobic wettabilities, J. MATER. PROCESS. TECH. 260 (2018) 1-8.

[22] S.C. Vlădescu, S. Medina, A.V. Olver, Lubricant film thickness and friction force measurements in a laser surface textured reciprocating line contact simulating the piston ring - liner pairing, TRIBOL. INT. 98 (2016) 317-329.

[23]D.S. Xiong, Y.K. Qin, J.L. Li, Tribological properties of PTFE/laser surface textured stainless steel under starved oil lubrication, TRIBOL. INT. 82 (2015) 305-310.

[24] A. Gherca, A. Fatu, M. Hajjam, Influence of surface texturing on the hydrodynamic performance of a thrust bearing operating in steady-state and transient lubrication regime, 
TRIBOL. INT. 102 (2016) 305-318.

2 [25] S. Wos, W. Koszela, P. Pawlus, Determination of oil demand for textured surfaces under

3 conformal contact conditions, TRIBOL. INT. 93 (2016) 602-613.

4 [26]Z.R. Zhang, X.W. Zhang, J.H. Yan, Manifold method coupled velocity and pressure for

5 Navier-Stokes equations and direct numerical solution of unsteady incompressible $6 \quad$ viscous flow, COMPUT. FLUIDS. 39 (2010) 1353-1365.

7 [27]L.V. Mirantsev, A.K. Abramyan, Couette flows between various bounding substrates, PHYS. LETT. A. 384 (2020) 126181. 
Figures

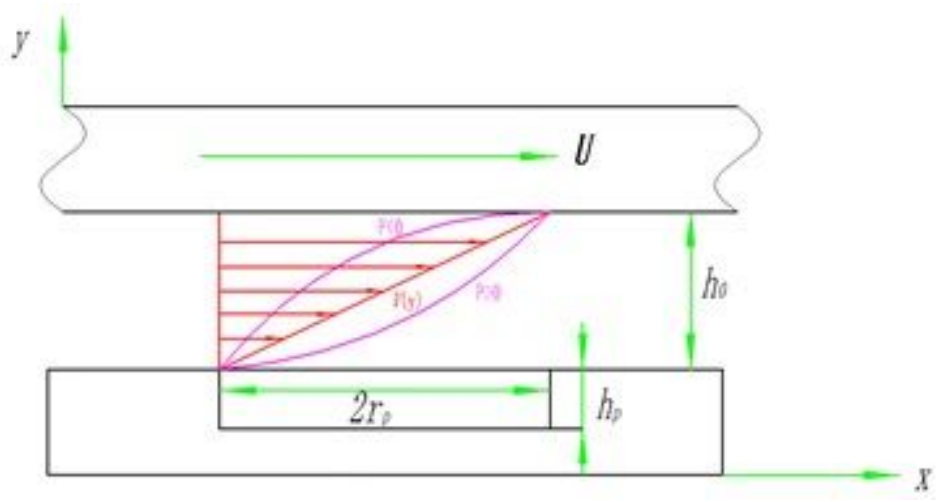

Figure 1

Couette flow

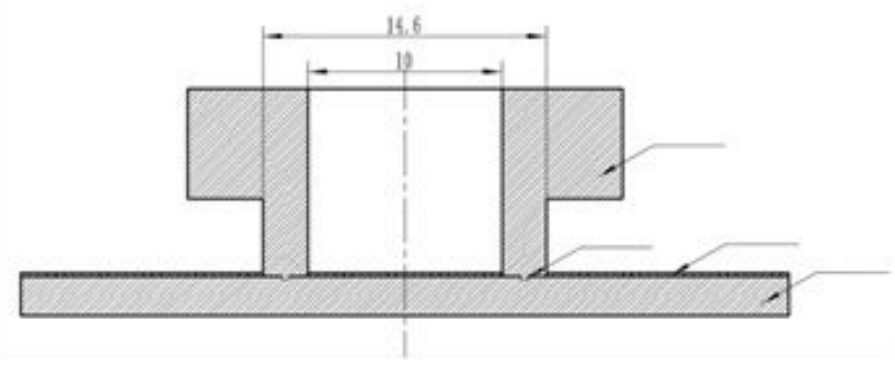

Figure 2

Couette flow

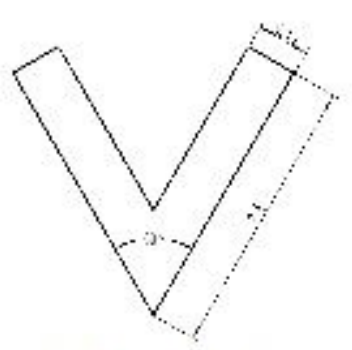

(a) chevron texture

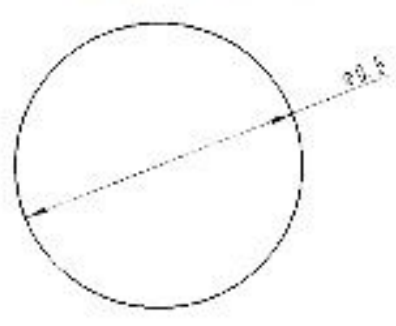

(c) circular texture

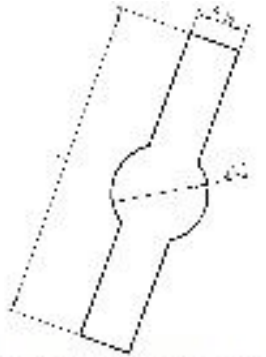

(b) composite texture

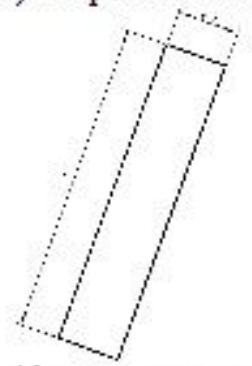

(d) groove tex ture

Figure 3 
The size of the texture

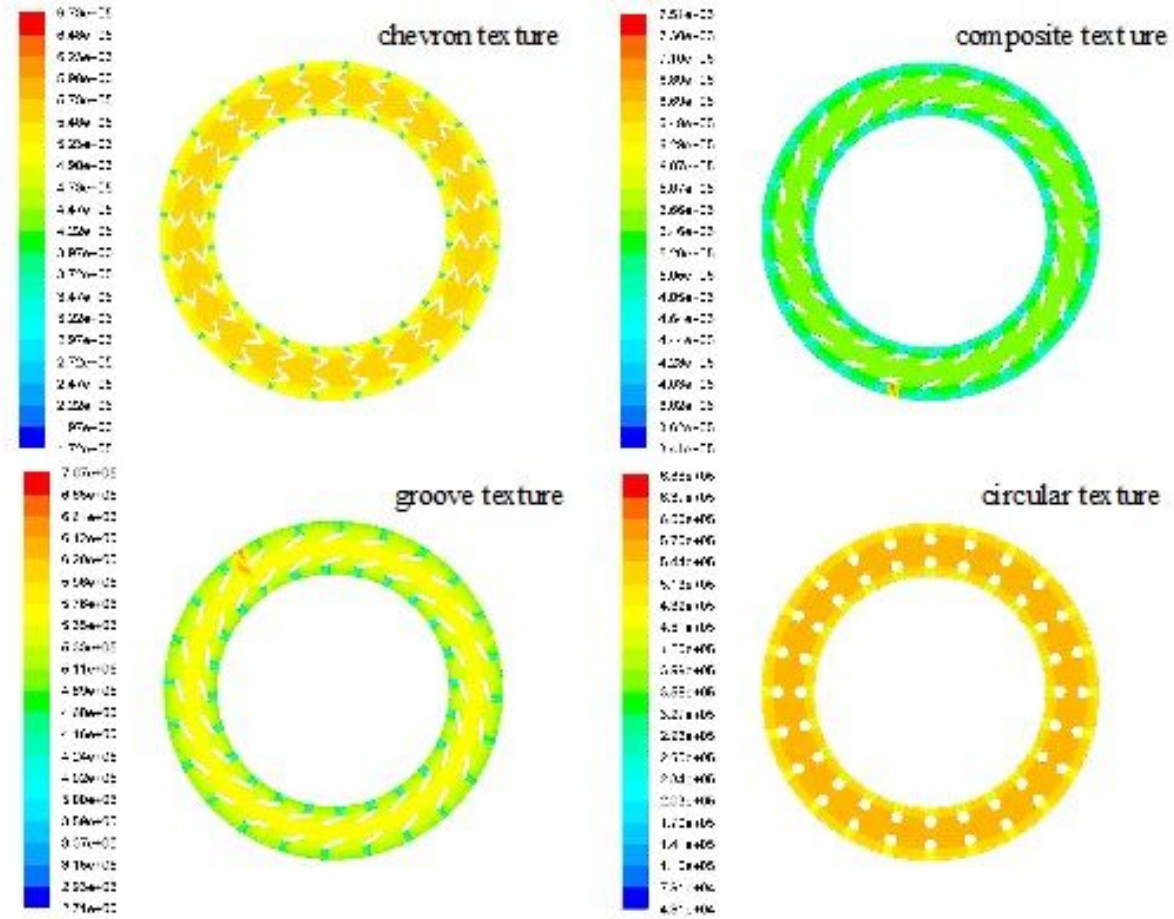

(a) Overall pressure doud diagram
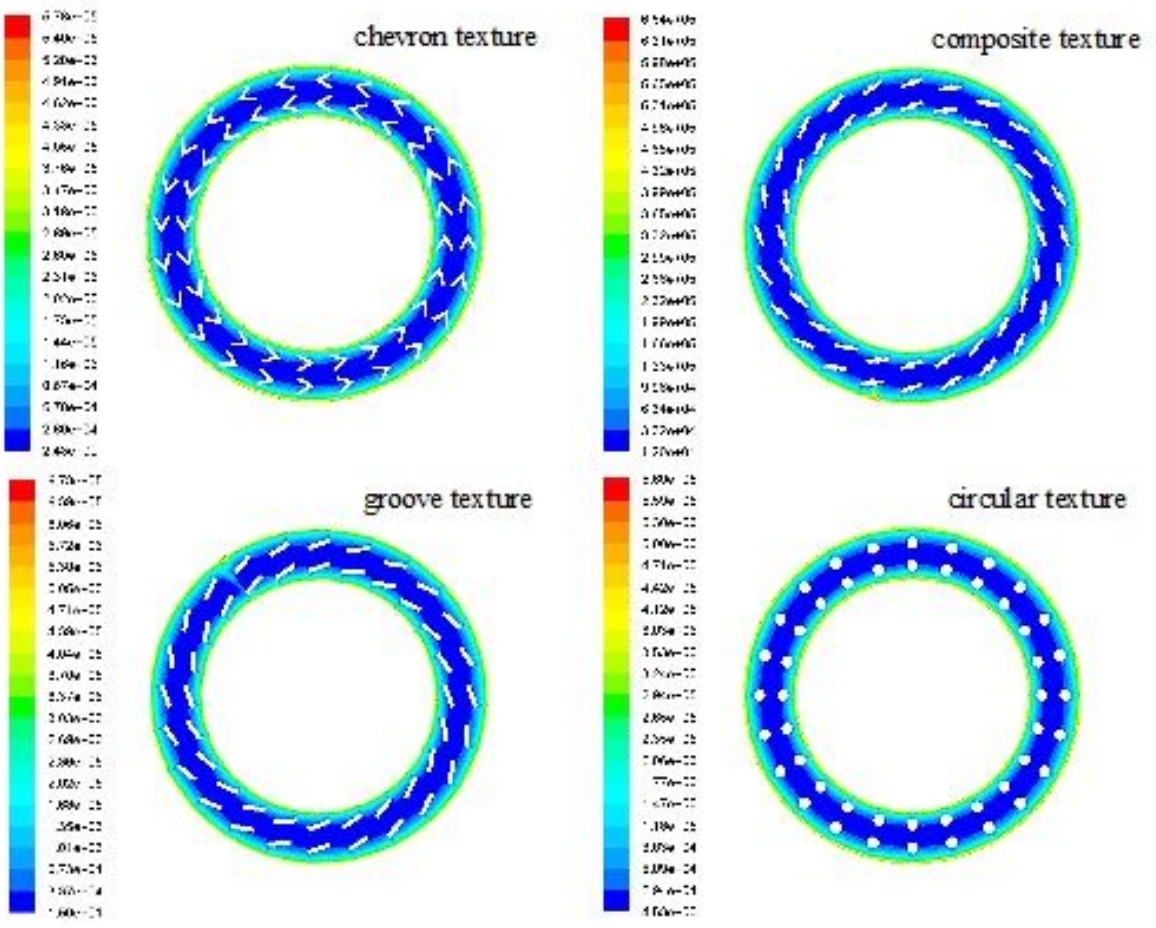

(b) Dynamic pressure cloud diagram

\section{Figure 4}

Overall pressure cloud diagram and dynamic pressure cloud diagram of overall texture 

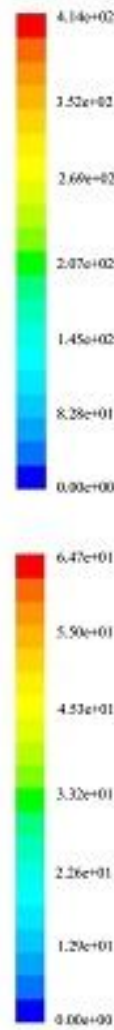
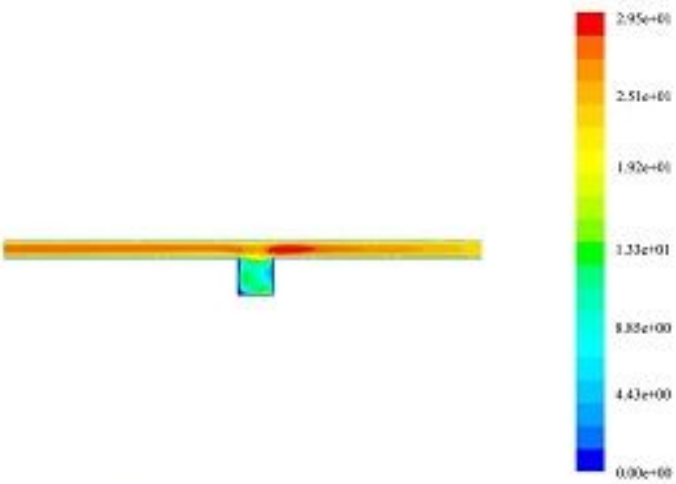

(a) chevron texture

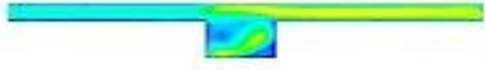

(c) groove tex ture

\section{Figure 5}

Cloud diagram of velocity distribution

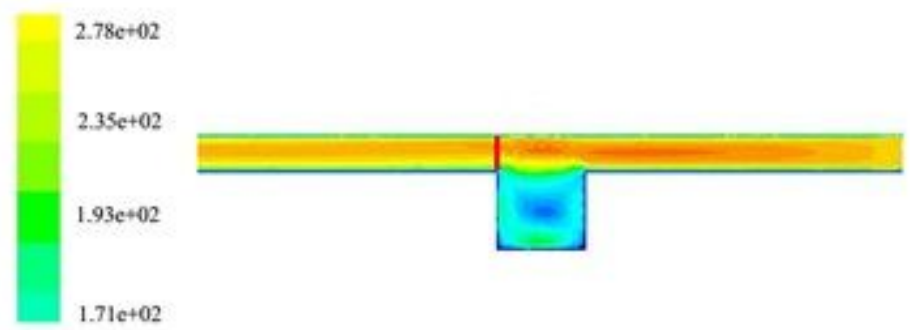

\section{Figure 6}

Schematic diagram of the location of the data selection line 


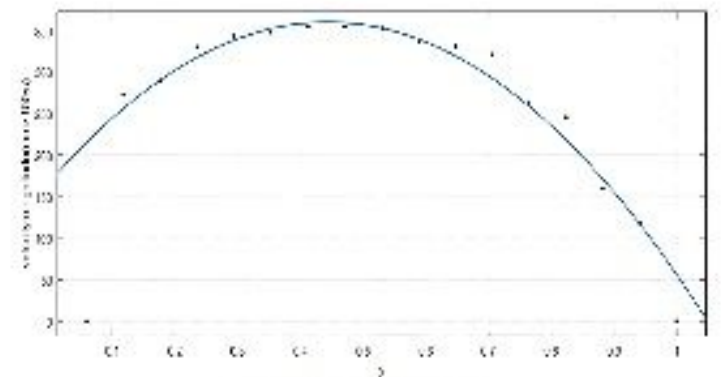

(a) chevron texture

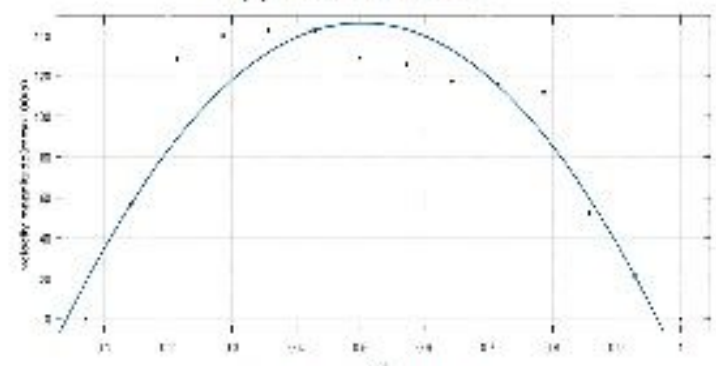

(c) groove texture

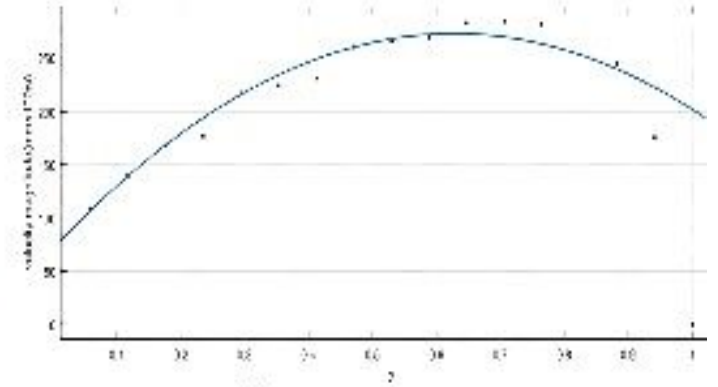

(b) composite texture

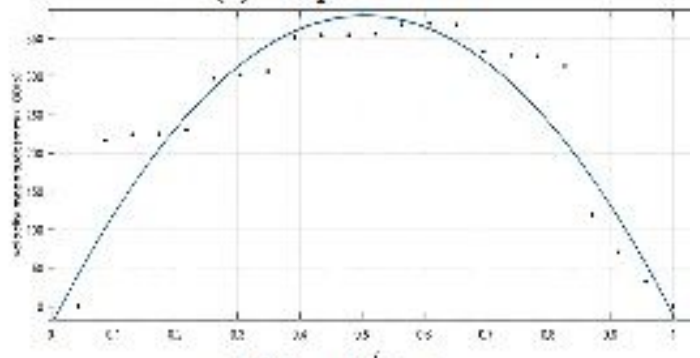

(d) circular texture

\section{Figure 7}

Fitting curves of four textures
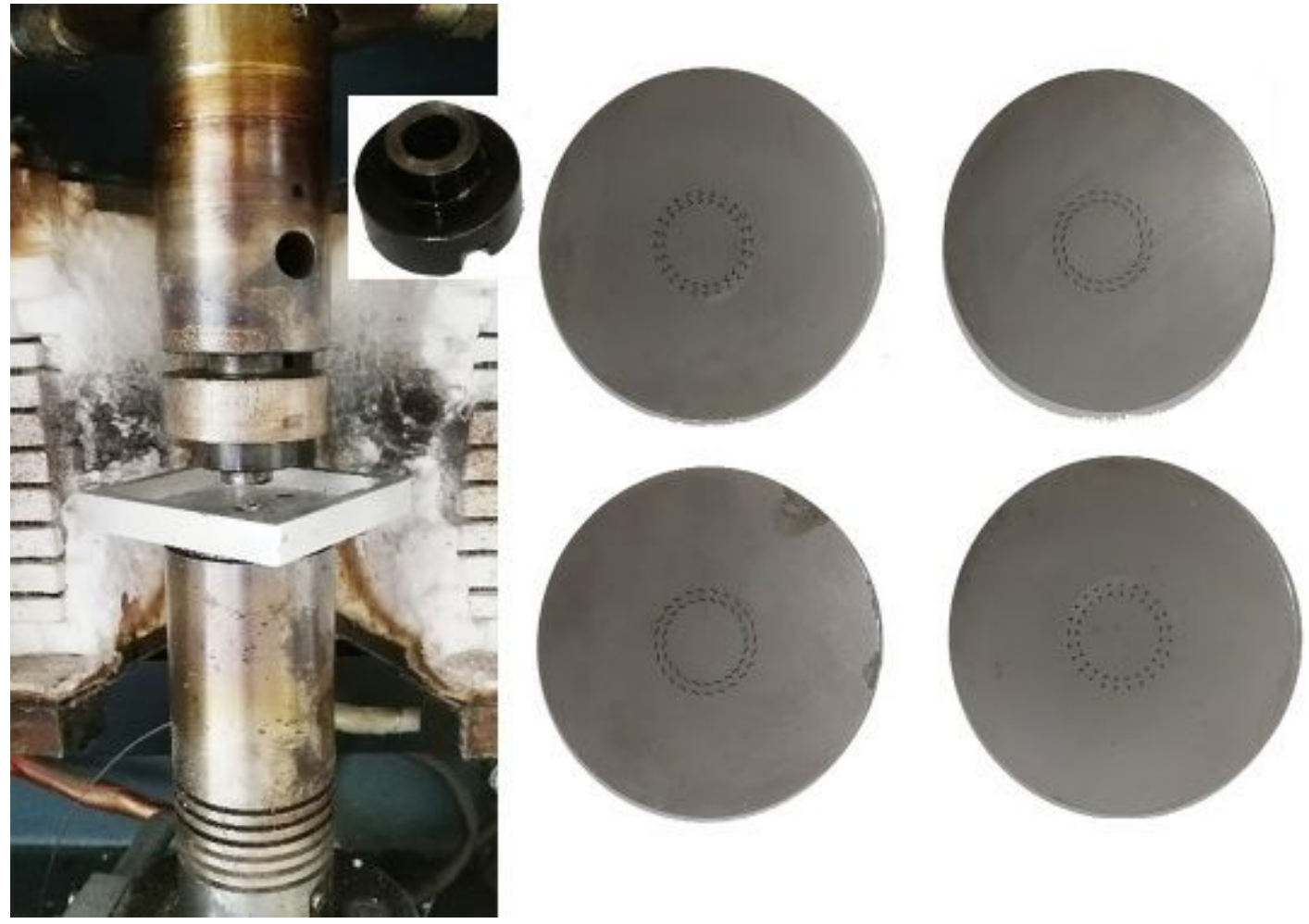

Figure 8

Friction and wear testing machine, 45 steel, and workpiece after processing texture 

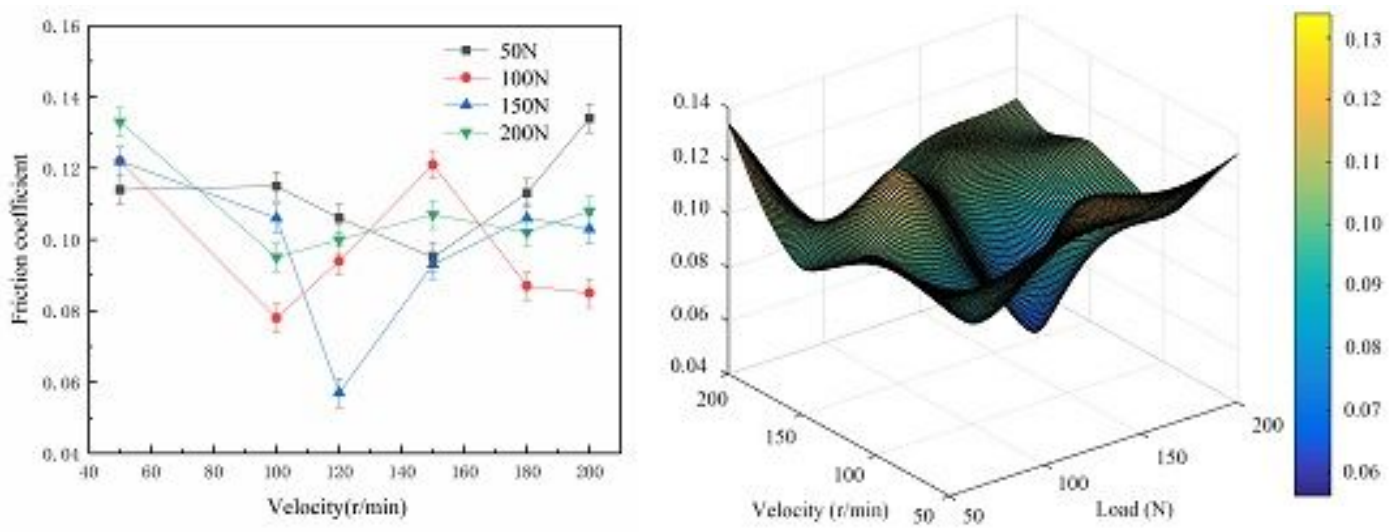

Figure 9

Experimental results of chevron texture
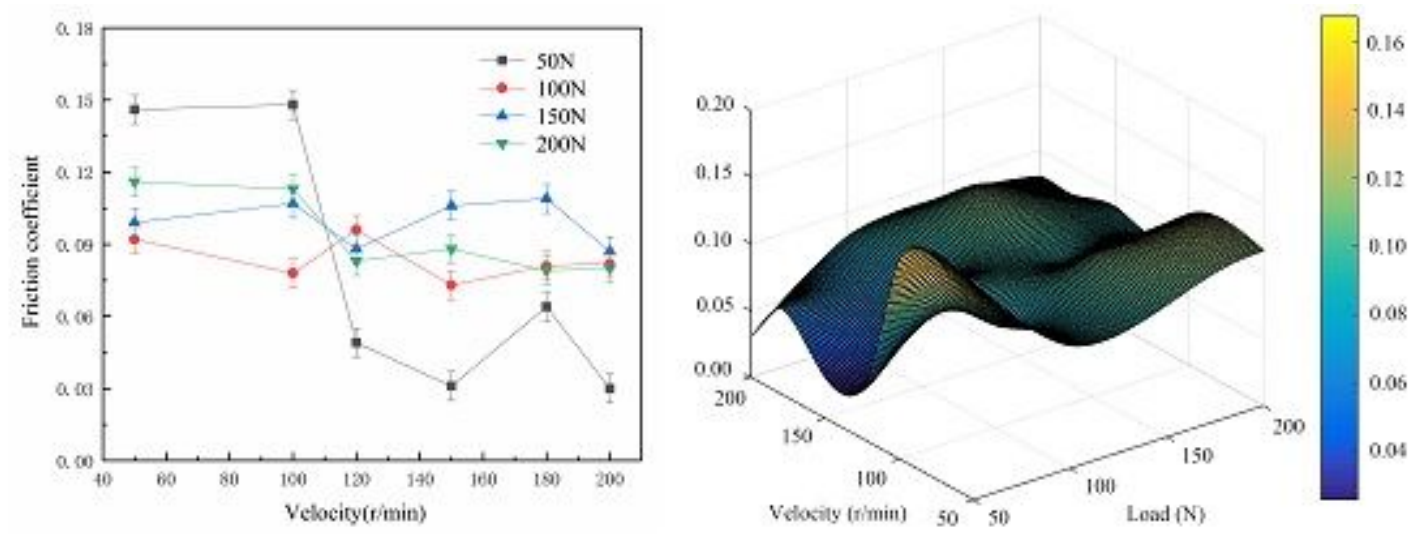

Figure 10

Experimental results of composite texture
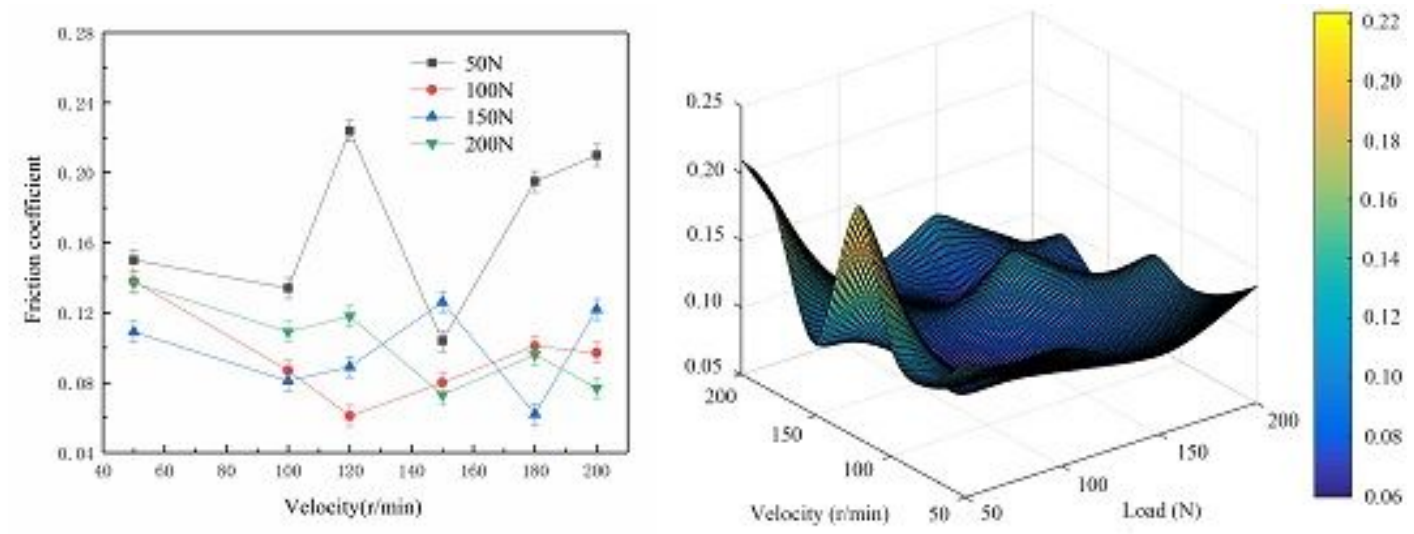

Figure 11

Experimental results of groove texture 

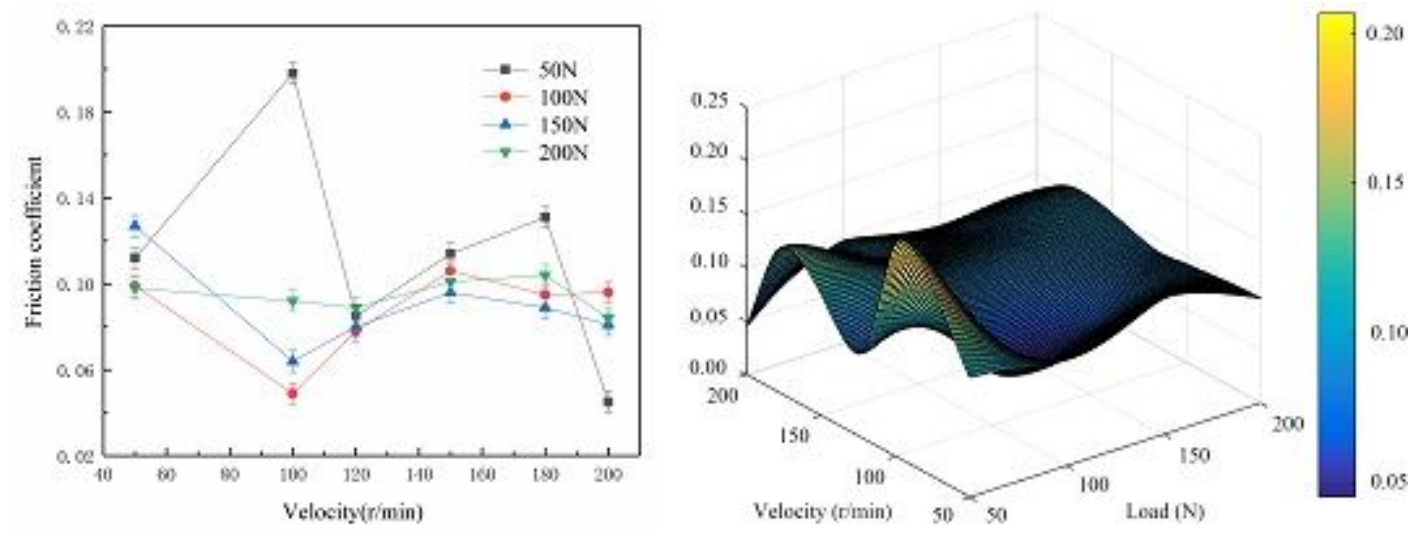

Figure 12

Experimental results of circular texture

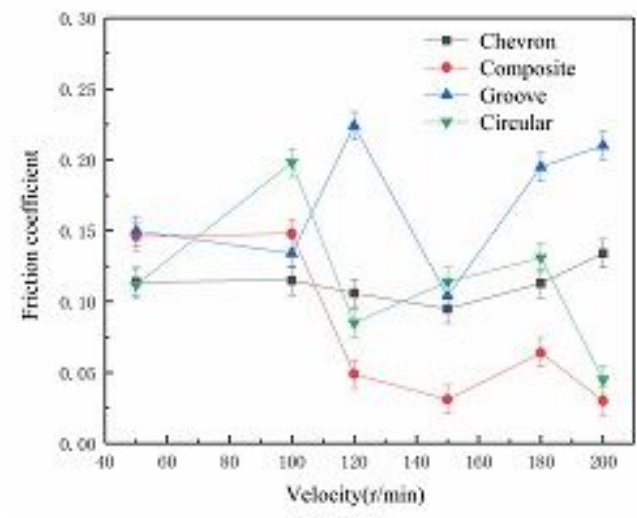

(a) $50 \mathrm{~N}$

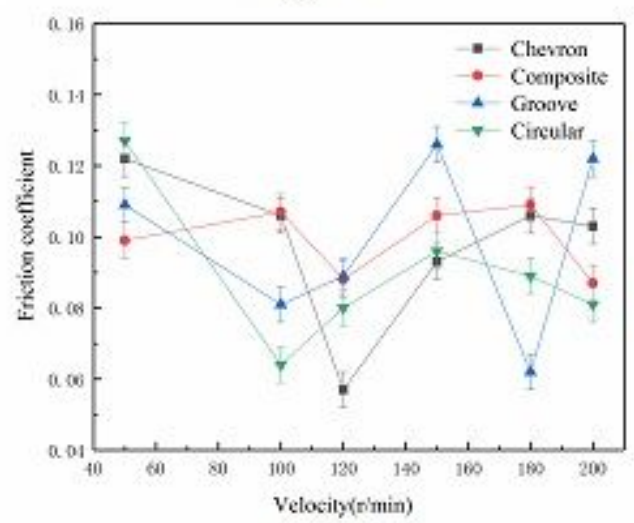

(c) $150 \mathrm{~N}$

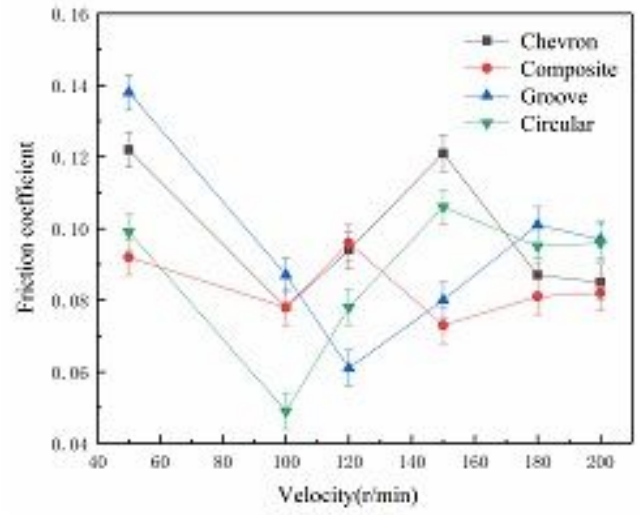

(b) $100 \mathrm{~N}$

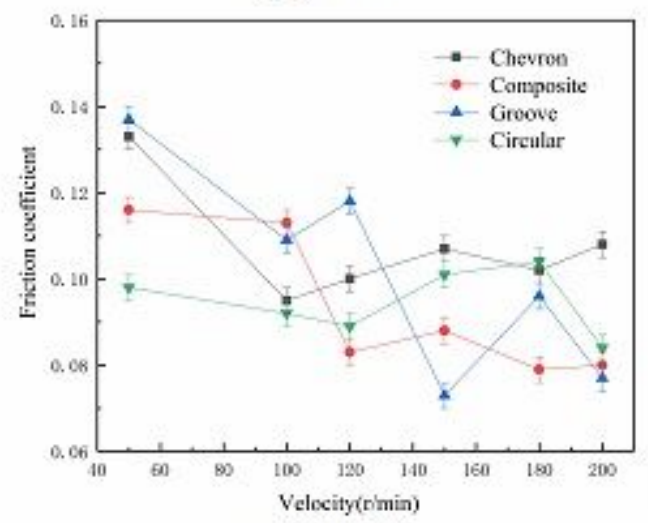

(d) $200 \mathrm{~N}$

Figure 13

Variation curve of friction coefficient of different textures under the same loading force 